\title{
The Synchronized and Integrated Two-Level Lot Sizing and Scheduling Problem: Evaluating the Generalized Mathematical Model
}

\author{
Claudio F. M. Toledo, ${ }^{1}$ Alf Kimms, ${ }^{2}$ Paulo M. França, ${ }^{3}$ and Reinaldo Morabito ${ }^{4}$ \\ ${ }^{1}$ Department of Computer Systems, Institute for Science Mathematics and Computing, Universidade de São Paulo, \\ 13566-590 São Carlos, SP, Brazil \\ ${ }^{2}$ Department of Technology and Operations Management, Mercator School of Management, University of Duisburg-Essen, \\ 47057 Duisburg, Germany \\ ${ }^{3}$ Department of Mathematics and Computer Science, Universidade Estadual Paulista, 19060-900 Presidente Prudente, SP, Brazil \\ ${ }^{4}$ Department of Production Engineering, Universidade Federal de São Carlos, 13565-905 São Carlos, SP, Brazil
}

Correspondence should be addressed to Reinaldo Morabito; morabito@ufscar.br

Received 4 December 2014; Revised 16 April 2015; Accepted 23 April 2015

Academic Editor: Joaquim Joao Judice

Copyright (C) 2015 Claudio F. M. Toledo et al. This is an open access article distributed under the Creative Commons Attribution License, which permits unrestricted use, distribution, and reproduction in any medium, provided the original work is properly cited.

\begin{abstract}
This paper presents the synchronized and integrated two-level lot sizing and scheduling problem (SITLSP). This problem is found in beverage production, foundry, glass industry, and electrofused grains, where the production processes have usually two interdependent levels with sequence-dependent setups in each level. For instance, in the first level of soft drink production, raw materials are stored in tanks flowing to production lines in the second level. The amount and the time the raw materials and products have to be stored and produced should be determined. A synchronization problem occurs because the production in lines and the storage in tanks have to be compatible with each other throughout the time horizon. The SITLSP and its mathematical model are described in detail by this paper. The lack of similar models in the literature has led us to also propose a set of instances for the SITLSP, based on data provided by a soft drink company. Thus, a set of benchmark results for these problem instances are established using an exact method available in an optimization package. Moreover, results for two relaxations proved that the modeling methodology could be useful in real-world applications.
\end{abstract}

\section{Introduction}

The problem studied in this paper is motivated by a real situation found in soft drink companies. In the bottling industry, production involves two interdependent levels with decisions about raw material storage in tanks and soft drink bottling in lines. In the first level, decisions regarding the amount and time the raw materials have to be stored in each one of the available tanks should be made. Similarly, in the second level, the lot size of each demanded item and its corresponding scheduling in each line should be determined. A capacitated lot sizing and scheduling problem has to be solved on each one of these two levels (Figure 1). This problem covers various issues of lot sizing and scheduling that have been dealt with on isolation only in the literature before. The challenging aspect is the combination of all these issues in one interdependent two-level problem. Capacitated lot sizing (and scheduling) is a topic of broad interest and has attracted many researchers. While capacitated lot sizing (and scheduling) is an NP-hard optimization problem $[1,2]$, the problem of finding a feasible solution is NP-complete already [3], if setup times are present.

One of the few optimal procedures for solving lot sizing and scheduling problems with sequence-dependent setup times can be found in Haase and Kimms [4]. Kovács et al. (2009) presented a more compact model based on the previous one proposed by Haase and Kimms [4], which allows solving larger instances within a reasonable computation time. Luche et al. [5] and Clark et al. [6] presented mixed 


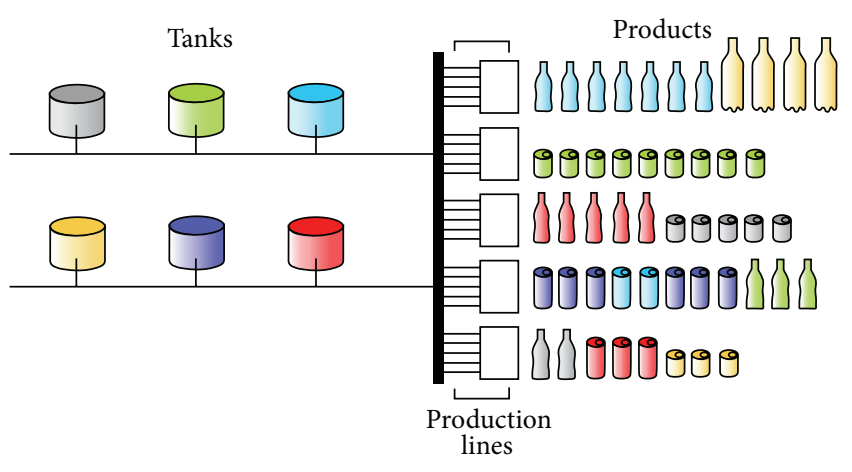

FIGURE 1: The two-level production process.

integer programming (MIP) models for lot sizing and scheduling in the presence of setup times for problems found in electrofused grain and animal nutrition industries. Concerning multilevel lot sizing problems, several publications exist (e.g., [7-10]). The present paper deals with a multilevel lot sizing and scheduling problem, which is difficult to tackle even if the capacity is unlimited $[11,12]$, and there are parallel machines represented by production lines (Figure 1). The work in de Matta and Guignard [13] deals with rolling production schedules for lot sizing with parallel machines. A study on sequence-dependent setup costs with parallel machines is found in Kang et al. [14]. A lot sizing problem in a semiconductor assembly with parallel machines was described by Kuhn and Quadt [15] and Quadt and Kuhn [16]. A GRASP heuristic with path-relinking intensification was applied by Nascimento et al. [17] to solve instances of the capacitated lot sizing problem with parallel machines.

A. R. Clark and S. J. Clark [18] proposed a MIP formulation for a lot sizing and scheduling problem on parallel machines with sequence-dependent setup times. Their complex MIP formulation was solved using approximate models in a rolling horizon basis with a reduced number of binary variables per period. Model reformulations were also presented by Wolsey [19] to make standard software applicable. Clark [20] presented a mathematical model for the planning of a canning line in a drinks manufacturer. He proposed approaches using local search integrated with the solution of approximate MIP models. Gupta and Magnusson [21] presented a model for the capacitated lot sizing and scheduling problem with sequence-dependent setup costs and nonzero setup times. The solver CPLEX was used to find optimal solutions for small sized instances. The authors also determined lower bounds for large sized instances using row aggregation and relaxing the integrality of some model variables. AlmadaLobo et al. [22] proposed models for the same problem tackled by Gupta and Magnusson [21], which were solved using specific-purpose heuristics and the CPLEX solver.

A synchronization problem arises in a two-level problem reported by Almada-Lobo et al. [23] and Toledo et al. [24]. Their focus is a glass container industry where the first level deals with the glass color produced in the furnaces and the second level deals with the color of the containers that are produced afterwards. MIP formulations are tailored to tackle this two-level and synchronized production planning problem where metaheuristic approaches are proposed. Baldo et al. [25] studied another two-level problem in the brewery industry: preparing the liquids including fermentation and maturation inside the fermentation tanks and bottling the liquids on the filling lines. The problem differs from soft drink problems mainly due to the relatively long lead times required for the fermentation and maturation processes and because the ready liquid can remain in the tanks for some time before being bottled. The surveys in Drexl and Kimms [26], Karimi et al. [27], Jans and Degraeve [28, 29], and Ramezanian et al. [30] can be referred to for complete overviews in lot sizing problems and industrial modeling for lot sizing problems.

A work that comes close to our case is the one described in Meyr [31], which is an extension of Meyr [32]. Meyr [31] considered a lot sizing and scheduling problem with nonidentical parallel machines, inventory costs, production costs, and sequence-dependent setup times and costs. However, the soft drink industry problem in the present paper is more complex in the sense that it has two lot sizing and scheduling problems, each of them similar to Meyr's problem, one for lines and another for tanks. The problem solution should find simultaneously the lot sizing and scheduling of raw materials in tanks and soft drinks in the bottling lines, because there is interdependence between these two levels, which is not present in Meyr's approach. Thus, new variables and constraints able to synchronize and integrate these two levels need to be added. There are studies dealing with supply chain synchronization or integration applied to decentralized manufacturers [33]. However, the synchronization aspect dealt with by the present paper is closer to the work of Tempelmeier and Buschkühl [34]. These authors introduced a synchronization problem in a multi-item multimachine lot sizing and scheduling problem. This problem is found in the production system of automobile suppliers. There is a predetermined assignment of products to machines where a common resource is necessary for setup processes on all machines.

Bearing in mind the mentioned aspects, the problem studied by the present paper is called synchronized and integrated two-level lot sizing and scheduling problem (SITLSP). Some works related with the specific soft drink industrial situation considered by the SITLSP have been published recently. For instance, in Ferreira et al. [35], a simplified formulation was presented for the SITLSP, where each bottling line has a dedicated tank and each tank can be filled with all liquid flavors needed by this line. Single-stage formulations were presented in Ferreira et al. [36] based on the general lot sizing and scheduling problem (GLSP) and on the asymmetric travelling salesman problem (ATSP). The solutions found using these formulations outperformed those found by solving the formulation described in Ferreira et al. [35]. Toledo et al. [37] introduced a genetic algorithm/mathematical programming approach using Ferreira et al.s [35] model, which reached competitive or better solutions for the real-world instances solved in Ferreira et al. [36]. All these recent results assume the hypotheses of dedicated tanks. In our formulation, there are no dedicated tanks, as it will be explained later. 
There are no existing modeling approaches to be directly applicable that include all the issues simultaneously considered: lot sizing, scheduling, setup (time and cost), parallel machines, among others. Thus, formulating a specific model seems to be in order. In Toledo et al. [38] we have already presented a related formulation of this paper (in Portuguese) in a very compact form, but now we spend a lot of effort to explain all the model constraints, providing details about their meanings and interpretations. An effective method to deal with all the aspects considered by the SITLSP was first proposed by Toledo et al. [39]. This method based on a multipopulation genetic algorithm with individuals hierarchically structured in trees was able to solve instances with several levels of difficulty based on data provided by a soft drink industry and the solutions were compared with AMPL/CPLEX solutions reported on that paper.

To sum up, the main contributions of this paper are to present in detail a mathematical formulation for the SITLP, to evaluate its performance solving real-world problem instances, and to define sets of benchmark instances for the SITLSP. A mathematical model serves many purposes as to provide an unequivocal definition of the problem better than a textual description can do. The present paper combines ideas from the general lot sizing and scheduling problem (GLSP) with the continuous setup lot sizing problem (CSLP) [26] to describe the SITLSP. The model is coded next and sets of small (artificially created) instances are solved using commercial software packages to find optimum solutions which can be used, for example, as benchmark to evaluate heuristics proposed for the same problem. Finally, medium-to-large instances are created and solved, applying some relaxation over the formulation when the optimal or even a feasible solution can be found by the exact method.

In the next section, we describe the industrial problem in detail with some examples that will help us to explain many specific problem issues. In the section on Mathematical Models, a MIP model is presented for the SITLSP. In section on Computational Results, we show some computational results obtained by solving the model with the modeling language AMPL in combination with the CPLEX solver. Finally, in Conclusions we present concluding remarks and discuss perspectives for future research.

\section{The Industrial Problem}

To make the explanation easier, the problem is posed as a soft drink industrial problem. Taking this into account, what it is referred to as product in the problem is the combination of the soft drink flavor and the type of container. Each of the soft drinks is available in one or more bottle types, such as glass bottles, plastic bottles, cans or bag-in-boxes of different sizes. In general, only a subset of the bottling lines is capable of producing a certain product, and products can be produced using production lines in parallel. Various products share a common production line, which cannot produce more than one product at a time. However, whenever the product switches, a setup time (of up to several hours) is required to prepare the production line for the next product. Production lines can maintain the setup state. When the production of a product is preempted for a while and continued after some idle time, then no setup is required. Setup times are sequencedependent, meaning that the order in which products are produced affects the required time to perform a setup.

Let us take an example considering a time horizon of 2 periods for a production process with 5 raw materials, 6 products, 3 tanks, and 3 lines. Each time period has 5 time units of capacity. Suppose the following:

(i) $\operatorname{Rm} A$ (raw material $A$ ) produces $P 1$ (product 1 ).

(ii) $\mathrm{Rm} B$ produces $P 2$ and $P 3$.

(iii) $\mathrm{RmC}$ produces $P 4$.

(iv) $\mathrm{RmD}$ produces $P 5$.

(v) $\mathrm{RmE}$ produces $P 6$.

(vi) $L 1$ (line 1) can bottle $P 1, P 2$, and $P 3$.

(vii) $L 2$ can bottle $P 1, P 4$, and $P 5$.

(viii) $L 3$ can bottle $P 1$ and $P 6$.

(ix) $\mathrm{Tk} 1$ (tank 1) can store $\operatorname{Rm} A, \operatorname{Rm} B$, and $\mathrm{RmC}$.

(x) Tk2 can store $\operatorname{Rm} A, \operatorname{Rm} B, \operatorname{Rm} C$, and $\operatorname{Rm} D$.

(xi) $\mathrm{Tk} 3$ can store $\mathrm{RmE}$.

In the first time period, the demands of products are 100 units of $P 1, P 4$, and $P 6$ and 50 units of $P 5$. In the second time period, the demands are 100 units of $P 2$ and $P 3$ and 50 units of $P 4$ and $P 5$. We are mentioning only relevant data for this example. Figure 2 shows a possible schedule for a production line level taking into account the line constraints as explained before.

The processing time for each product to be produced is shown in Figure 2. The assignments satisfied the set of products allowed to be produced in each line and the demands in each period. There are also sequence-dependent setup times from product $P 2$ to $P 3$ in $L 1$ and from $P 4$ to $P 5$ in $L 2$. This schedule only pays attention to the line constraints, but it does not take into account the tank constraints discussed next.

The raw material (i.e., the soft drink) that is bottled in a production line comes from a storage tank with limited storage capacity. Different soft drinks cannot be put in a tank simultaneously. Hence, from time to time a tank must be filled with a particular soft drink, which might be another drink or the same as before. Nevertheless, whenever a tank is filled, a significant (sequence-dependent) setup time occurs to clean and fill the tank, even if the same soft drink is filled into the tank as before. For technical reasons, a tank can only be filled up when it is empty. During the time of filling up a tank, nothing can be pumped to a production line from that tank. There is no fixed assignment between tanks and production lines. Every tank can be connected to every production line and a tank can feed more than one production line.

In the previous example, let us assume that all tanks have $100 \mathrm{~L}$ (liters) of maximum storage capacity. Suppose that $2 \mathrm{~L}$ of $\mathrm{Rm} A$ is necessary to produce $1 \mathrm{u}$ (unit) of $P 1,0.5 \mathrm{~L}$ of $\mathrm{Rm} B$ for $1 \mathrm{u}$ of $P 2$ and $1 \mathrm{u}$ of $P 3,1 \mathrm{~L}$ of $\mathrm{RmC}$ for $P 4,2 \mathrm{~L}$ of $\mathrm{Rm} D$ for $P 5$, and $1 \mathrm{~L}$ of $\mathrm{RmE}$ for $P 6$. Figure 3 shows a possible schedule considering the tank constraints. 


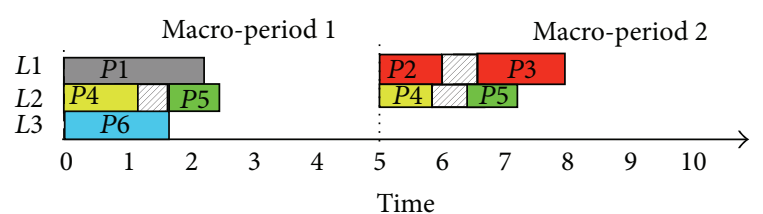

FIGURE 2: Schedule for the production line level.

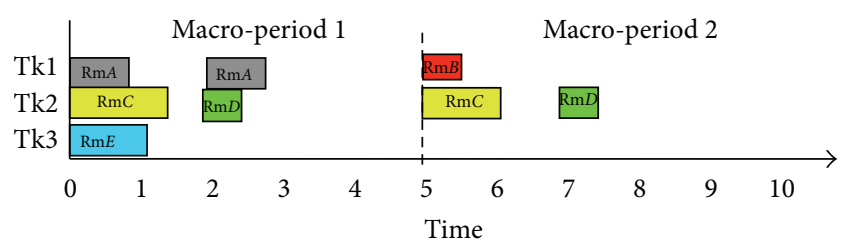

FIgURE 3: Schedule for tank level.

A total of $200 \mathrm{~L}$ of $\mathrm{Rm} A$ to produce $100 \mathrm{u}$ of $P 1$ is necessary. Therefore, tank Tk1 must be refilled with $\mathrm{Rm} A$ and the setup times are shown in Figure 3. The interdependence between the two levels of the problems becomes clear now. The schedule in Figure 3 cannot be created without knowing the line schedule in Figure 2. For instance, it is necessary to know when $100 \mathrm{u}$ of $P 1$ are scheduled on $L 1$ to fill Tk1 with $\mathrm{Rm} A$. This also happens in Tk2 where it is necessary to know when $P 4$ is scheduled on $L 2$ to fill Tk2 with $\mathrm{RmD}$. The schedules proposed in Figures 2 and 3 cannot be executed without repairs. The same problem would happen, if we had scheduled the tank level first. Figure 4 illustrates a possible repair that swapped product $P 4$ for $P 5$ from the schedules in Figures 2 and 3.

Bottling is usually done to meet a given demand (per week) with a production planning horizon of four weeks in particular soft drink companies. The decision maker has to find out how many units of what product should be produced when and on what production line. To be able to do so, the tanks must be filled appropriately. The objective can include the minimization of the total sum of setups, inventory holding and production costs. Thus, there is a multilevel (two level) lot sizing and scheduling problem with parallel processors (tanks on the first level and lines on the second), capacity constraints, and sequence-dependent setup times and costs. The mathematical model proposed by this paper in the next section has to integrate these two lot sizing and scheduling problems. The production in lines and the storage in tanks must be compatible with each other.

\section{A Model Formulation}

This section introduces the mathematical model formulation for the SITLSP. First, the basic ideas used by our formulation are described. Next, the objective function as well as each one of the model constraints is explained. Finally, an example is used to show the type of information returned by the model solution.

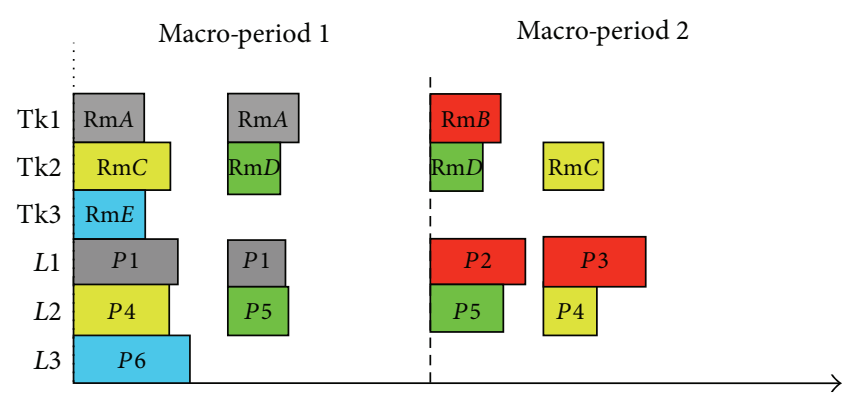

Figure 4: Occupation for tank and line slots.

3.1. Basic Ideas. The underlying idea for modeling our application combines issues from the general lot sizing and scheduling problem (GLSP) and the so-called continuous setup lot sizing problem (CSLP) (see [26] for a comparison of these models). A set of additional variables and constraints are also included in the model, which can describe the specific issues of the SITLSP.

From the GLSP, we adopt the idea of defining a fixed number ( $S$ for the production lines and $\bar{S}$ for the tanks) of slots per (macro-) period, so that at most one product or raw material can be scheduled per slot. What needs to be determined is for what product or raw material, respectively, a particular slot should be reserved in lines and tanks, and what lot size (a lot of size zero is possible) should be effectively scheduled, given a valid slot reservation. Let us take the schedule shown in Figures 2 and 3. Suppose now 2 macro-periods with $S=\bar{S}=2$ slots per period. Figure 4 shows only the simultaneous slot reservation for lines and tanks in each period.

The number of products in lines and raw materials in tanks cannot be greater than the total number of slots $(S=\bar{S}=$ 2 ) in each macro-period. There is not a full slot occupation in Figure 4. For instance, only one slot is effectively occupied by raw materials and products, respectively, in tank Tk3 and line $L 3$ during the first macro-period. In the second macroperiod, there is no slot occupied in Tk3 and L3. Following the GLSP idea, these two slots are reserved (P6 in line $L 3$ and $\mathrm{Rm} E$ in tank Tk3), but nothing is produced.

The use of slots in the model enables us to describe lines and tank occupation using variables and constraints indexed by slots. Therefore, we can define decision variables like $x_{j l s}=$ 1 , if slot $s$ in line $l$ can be used to produce product $j, 0$, otherwise; and $u_{l s}=1$, if a positive production amount is effectively produced in slot $s$ in production line $l(0$, otherwise). In the same way, we have decision variables like $\bar{x}_{j k s}=1$, if slot $s$ can be used to fill raw material $j$ in tank $k, 0$, otherwise; and $\bar{u}_{k s}=$ 1 , if slot $s$ is used to fill raw material in tank $k, 0$, otherwise. These variables (and others) will define constraints for the correct line (or tank) occupation, the reservation of only one product (raw material) per slot, and the effective production (storage) of products (raw materials) (see model constraints (2) -(10) and (30)-(39) in the next subsections).

In the SITLSP, the slots scheduled on one level with the slots scheduled on the other level need to be coordinated. For instance, a production line level, which must wait for a setup time on the tank level, requires the tank setup time to 
TABLE 1: Guideline for line and tank constraints.

\begin{tabular}{|c|c|c|}
\hline Line constraints & Tank constraints & Meaning \\
\hline$(2)$ & $(30)$ & Assignments not allowed for lines and tanks \\
\hline (3) & $(31)$ & Assignments of products or raw materials to slots \\
\hline$(4),(16)$, and (17) & $(32)-(34)$ & $\begin{array}{l}\text { Link between the products or raw material assignments and the respective lot size } \\
\text { produced or stored. }\end{array}$ \\
\hline (7) & (35) and (36) & Products or raw materials changeover. \\
\hline (8) and (9) & (37) and (38) & Changeover setup time \\
\hline (10) & (39) & Macro-period capacity \\
\hline (5) and (6) & (64) and (65) & Inventory balance \\
\hline$(11)-(13)$ & $(41)-(43)$ & Available capacity between endings of two consecutive slots. \\
\hline (14) and (15) & $(44)-(47)$ & Define only one beginning and only one ending for each slot. \\
\hline$(18)-(21),(23)-(24)$ & $(51)-(57),(59)-(61)$ & Define the beginning and the ending for a slot effectively used or not. \\
\hline (22) & $(58)$ & Define an order for slot occupation \\
\hline (25), (28), and (29) & (40), (48), (64), and (65) & Synchronize when a slot of raw material is taken from tanks and produced by lines. \\
\hline (26) and (27) & - & Use of the capacity of the first micro-period of each slot. \\
\hline- & (62), (63), and (65) & Refilling of tanks \\
\hline
\end{tabular}

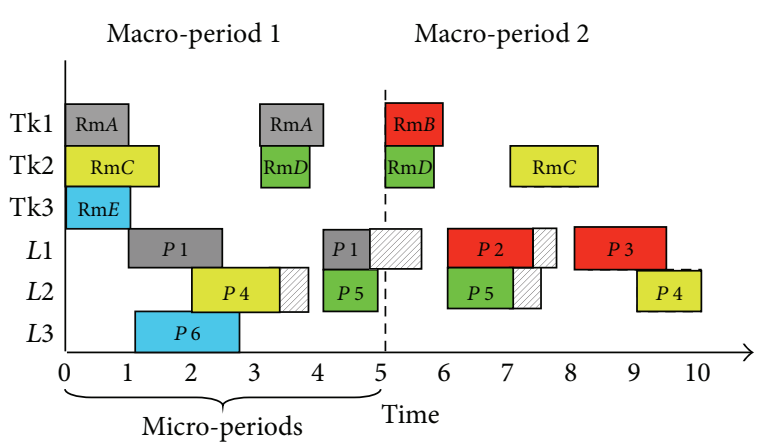

FIGURE 5: Synchronization between slots in lines and tanks.

be ready at least one small time unit before the production to start in the line level. For that reason, we introduce small micro-periods in a CSLP-like manner. The macro-periods are divided into a fixed number of small micro-periods with the same capacity. This capacity can be partially used, but the same micro-period cannot be occupied by two slots. Let us illustrate this idea in Figure 5, which synchronizes lines and tanks from Figure 4.

Each macro-period was divided into 5 micro-periods with the same length. In the first period, the production of $P 1, P 4$, and $P 6$ must start after the initial setup time of $\mathrm{Rm} A, \mathrm{RmC}$, and $\mathrm{Rm} E$, respectively. The raw material must be previously ready in the tank so that the production begins in lines. The setup time needed to refill Tk1 with $\operatorname{Rm} A$ and Tk2 with $\mathrm{Rm} D$ leads to an interruption of $P 1$ and $P 5$ production in lines $L 1$ and $L 2$, respectively. In the second period, there is an idle time in $L 2$ after $P 5$. Line $L 2$ is ready to produce $P 4$ after its setup time, but the production has to wait for the $\mathrm{RmC}$ setup time in $\mathrm{Tk} 2$.

Variables and constraints indexed by slots and microperiods will enable us to describe these situations. Binary variables $\bar{x}_{l s \tau}^{B}$ and $\bar{x}_{l s \tau}^{E}$ indicate the micro-period $\tau$ when the production of slot $s$ begins or when it ends in line $l$, respectively. The analogous variables $\bar{x}_{k s \tau}^{B}$ and $\bar{x}_{k s \tau}^{E}$ are used to indicate micro-period $\tau$ on slot $s$ of tank $k$. It is also necessary to define linking variables $q_{k j l s \tau}$, which determine the amount of product $j$ produced in line $l$ in micro-period $\tau$ on slot $s$, using raw material from tank $k$. These variables (and others) establish constraints which link production lines and tanks and synchronize raw material and product slots (see model constraints (25)-(27), (40), and (64) in the next subsections).

The model constraints are formally defined in the next subsections. Table 1 has already separated them according to the meaning, making it easier to understand.

3.2. Objective Function. Suppose that we have $\bar{J}$ raw materials and $J$ products, $L$ production lines, and $\bar{L}$ tanks. The planning horizon is subdivided into $T$ macro-periods. For the modeling reasons explained before, we assume that at most $S$ slots per macro-period can be scheduled on each production line, and at most $\bar{S}$ slots per macro-period and per tank can be scheduled. These slot values can be set to sufficiently large numbers so that this assumption is not restrictive for practical purposes. Furthermore, for the same modeling reasons explained before, we assume that each macro-period is subdivided into $T^{m}$ micro-periods. The notation being used in the model description is summarized in the appendix.

As usual in lot sizing models, the objective is to minimize the total sum of setup costs, inventory holding costs and production costs. The first three terms in expression (1) represent setup, holding, and production costs for production lines and the next three the same costs for tanks. Notice that a feasible solution, which fulfills all the demand right in time without violating all constraints to be described, may not exist. Hence, we allow that $q_{j}^{0}$ units of the demand for product $j$ may not be produced to ensure that the model can always be solved (last term in (1)). Of course, a very high penalty $M$ is attached to 
such shortages so that whenever there is a feasible solution that fulfills all the demands, we would prefer this one:

$$
\begin{aligned}
& \sum_{i=1}^{J} \sum_{j=1}^{J} \sum_{l=1}^{L} \sum_{s=1}^{T \cdot S} s_{i j l} z_{i j l s}+\sum_{j=1}^{J} \sum_{t=1}^{T} h_{j} I_{j t}+\sum_{j=1}^{J} \sum_{l=1}^{L} \sum_{s=1}^{T \cdot S} v_{j l} q_{j l s} \\
& +\sum_{i=1}^{\bar{J}} \sum_{j=1}^{\bar{J}} \sum_{k=1}^{\bar{L}} \sum_{s=1}^{T \cdot \bar{S}} \bar{s}_{i j l} \bar{z}_{i j l s}+\sum_{j=1}^{\bar{J}} \sum_{k=1}^{\bar{L}} \sum_{t=1}^{T} \bar{h}_{j} \bar{I}_{j k, t \cdot T^{m}} \\
& +\sum_{j=1}^{\bar{J}} \sum_{k=1}^{\bar{L}} \sum_{s=1}^{T \cdot \bar{S}^{\prime}} \bar{v}_{j l} \bar{q}_{j k s}+M \sum_{j=1}^{J} q_{j}^{0} .
\end{aligned}
$$

3.3. Production Line: Usual Constraints. First, constraints for the production line level are presented which are usually found in lot sizing and scheduling problems:

$$
\begin{aligned}
& x_{j l s}=0 \\
& j=1, \ldots, J ; l \in\{1, \ldots, L\} \backslash L j ; s=1, \ldots, T \cdot S, \\
& \sum_{j=1}^{J} x_{j l s}=1 \quad l=1, \ldots, L ; s=1, \ldots, T \cdot S, \\
& p_{j l} q_{j l s} \leq C x_{j l s} \\
& j=1, \ldots, J ; l=1, \ldots, L ; s=1, \ldots, T \cdot S, \\
& I_{j 1}=I_{j 0}+q_{j}^{0}+\sum_{l=1}^{L} \sum_{s=1}^{S} q_{j l s}-d_{j 1} \quad j=1, \ldots, J \\
& I_{j t}=I_{j, t-1}+\sum_{l=1}^{L} \sum_{s=(t-1) S+1}^{t \cdot S} q_{j l s}-d_{j t} \\
& j=1, \ldots, J ; t=2, \ldots, T \text {, } \\
& z_{i j l s} \geq x_{j l s}+x_{i l, s-1}-1 \\
& i, j=1, \ldots, J ; l=1, \ldots, L ; s=1, \ldots, T \cdot S, \\
& \sigma_{l s}=\sum_{i=1}^{J} \sum_{j=1}^{J} \sigma_{i j l}^{\prime} z_{i j l s} \\
& i, j=1, \ldots, J ; l=1, \ldots, L ; s=1, \ldots, T \cdot S, \\
& \omega_{l t} \leq \sigma_{l,(t-1) S+1} \quad l=1, \ldots, L ; t=1, \ldots, T ; \\
& \sum_{s=(t-1) S+1}^{t \cdot S}\left(\sigma_{l s}+\sum_{j=1}^{J} p_{j l} q_{j l s}\right) \leq C+\omega_{l t} \\
& l=1, \ldots, L ; t=1, \ldots, T .
\end{aligned}
$$

A particular product can be produced on some lines, but not on all in general (2) and only one product $j$ is produced in each slot $s$ of line $l(3)$. The available time per macro-period is an upper bound on the time for production (4). The inventory balancing constraints (5) and (6) ensure that all demands are met taking into account the dummy variable $q_{j}^{0}$. Constraint
(7) defines the setup times $\left(z_{i j l s}\right.$ indicates this) based on slot reservations $\left(x_{j l s}\right)$. Thus, it is possible to determine the setup time $\sigma_{l s}$ to be included just in front of a certain slot (8). As a result, overlapping setup can happen and, for the first slot of a macro-period $t$, some setup time $\omega_{l t}$ may be at the end of the previous macro-period (9). The capacity $C$ in constraint (10) has to be incremented by $\omega_{l t}$. Thus, the total sum of production and setup times in a certain macro-period must not exceed the macro-period capacity $C$ by constraint (10).

3.4. Production Line: Time Slot Constraints. The constraints showed next describe how to integrate the micro-period idea from CLSP and the slot idea from GLSP. The main point here is to define specific constraints for the SITLSP which describe when some slots in lines will be used throughout the time horizon

$$
\begin{aligned}
& t \cdot C-\sum_{\tau=(t-1) T^{m}+1}^{t \cdot T^{m}} C^{m} \tau x_{l, t \cdot S, \tau}^{E} \geq \omega_{l, t+1} \\
& l=1, \ldots, L ; t=1, \ldots, T-1 \\
& \sum_{\tau=(t-1) T^{m}+1}^{t \cdot T^{m}} C^{m} \tau x_{l s \tau}^{E}-\sum_{\tau=(t-1) T^{m}+1}^{t \cdot T^{m}} C^{m} \tau x_{l, s-1, \tau}^{E} \geq \sigma_{l s} \\
& +\sum_{j=1}^{J} p_{j l} q_{j l s} \\
& l=1, \ldots, L ; t=1, \ldots, T ; s=(t-1) S+1, \ldots, T \cdot S, \\
& \sum_{\tau=(t-1) T^{m}+1}^{t \cdot T^{m}} C^{m} \tau x_{l,(t-1) S+1, \tau}^{E} \geq(t-1) C+\sigma_{l,(t-1) S+1}-\omega_{l t} \\
& +\sum_{j=1}^{J} p_{j l} q_{j l,(t-1) S+1} \\
& l=1, \ldots, L ; t=1, \ldots, T ; s=1, \ldots, T \cdot S, \\
& \sum_{\tau=(t-1) T^{m}+1}^{t \cdot T^{m}} x_{l s \tau}^{E}=1 \\
& l=1, \ldots, L ; t=1, \ldots, T ; s=(t-1) S+1, \ldots, T \cdot S, \\
& \sum_{\tau=(t-1) T^{m}+1}^{t \cdot T^{m}} x_{l s \tau}^{B}=1 \\
& l=1, \ldots, L ; t=1, \ldots, T ; s=(t-1) S+1, \ldots, T \cdot S, \\
& \sum_{j=1}^{J} p_{j l} q_{j l s} \leq C u_{l s} \quad l=1, \ldots, L ; s=1, \ldots, T \cdot S, \\
& \varepsilon u_{l s} \leq \sum_{j=1}^{J} q_{j l s} \quad l=1, \ldots, L ; s=1, \ldots, T \cdot S, \\
& \sum_{\tau=(t-1) T^{m}+1}^{t \cdot T^{m}} \tau x_{l s \tau}^{E}-\sum_{\tau=(t-1) T^{m}+1}^{t \cdot T^{m}} \tau x_{l s \tau}^{B} \leq T^{m} u_{l s} \\
& l=1, \ldots, L ; t=1, \ldots, T ; s=(t-1) S+1, \ldots, T \cdot S,
\end{aligned}
$$




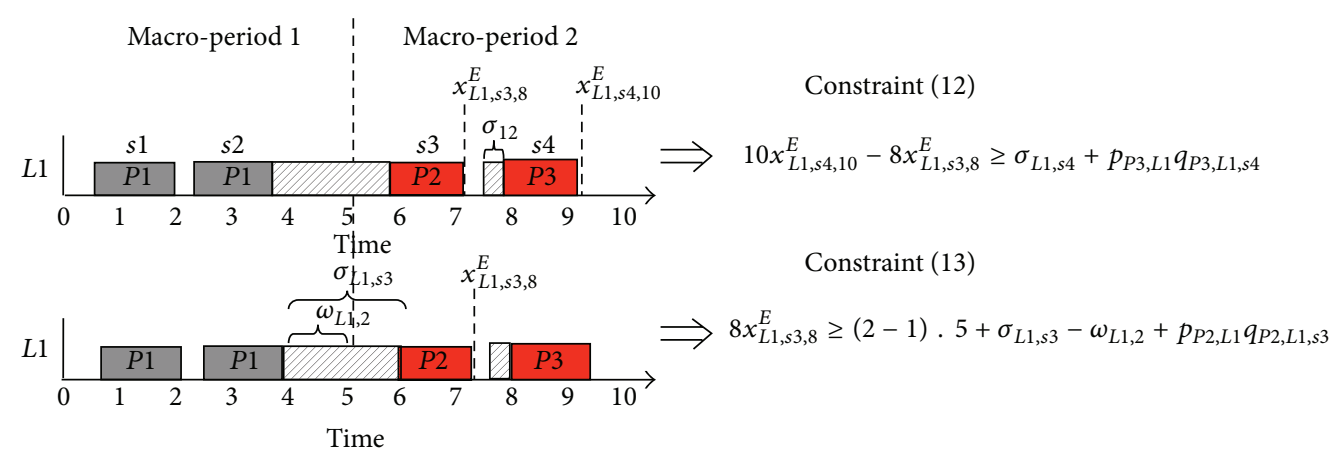

FIgURE 6: Keeping enough time between slots.

$$
\begin{aligned}
& \sum_{\tau=(t-1) T^{m}+1}^{t \cdot T^{m}} \tau x_{l s \tau}^{B}-\sum_{\tau=(t-1) T^{m}+1}^{t \cdot T^{m}} \tau x_{l s \tau}^{E} \leq u_{l s} \\
& l=1, \ldots, L ; t=1, \ldots, T ; s=(t-1) S+1, \ldots, T \cdot S, \\
& \sum_{\tau=(t-1) T^{m}+1}^{t \cdot T^{m}} \tau x_{l s \tau}^{B}-\sum_{\tau=(t-1) T^{m}+1}^{t \cdot T^{m}} \tau x_{l, s-1, \tau}^{E} \leq T^{m} u_{l s} \\
& \quad l=1, \ldots, L ; t=1, \ldots, T ; s=(t-1) S+2, \ldots, T \cdot S, \\
& \sum_{\tau=(t-1) T^{m}+1}^{t \cdot T^{m}} \tau x_{l,(t-1) S+1, \tau}^{B}-\left((t-1) T^{m}+1\right) \leq T^{m} u_{l,(t-1) S+1} \\
& \quad l=1, \ldots, L ; t=1, \ldots, T,
\end{aligned}
$$

$u_{l s} \geq u_{l, s+1}$

$l=1, \ldots, L ; t=1, \ldots, T ; s=(t-1) S+1, \ldots, T \cdot S-1$,

$$
\begin{aligned}
& \varepsilon u_{l s}+\sum_{\tau=(t-1) T^{m}+1}^{t \cdot T^{m}} C^{m} \tau x_{l s \tau}^{E} \\
& -\sum_{\tau=(t-1) T^{m}+1}^{t \cdot T^{m}} C^{m} \tau x_{l s \tau}^{B} \leq \sum_{j=1}^{J} p_{j l} q_{j l s} \\
& \quad l=1, \ldots, L ; t=1, \ldots, T ; s=(t-1) S+1, \ldots, T \cdot S, \\
& \sum_{\tau=(t-1) T^{m}+1}^{t \cdot T^{m}} C^{m} \tau x_{l s \tau}^{E}-\sum_{\tau=(t-1) T^{m}+1}^{t \cdot T^{m}} C^{m} \tau x_{l s \tau}^{B} \geq \sum_{j=1}^{J} p_{j l} q_{j l s} \\
& -C^{m} \quad \\
& \quad l=1, \ldots, L ; t=1, \ldots, T ; s=(t-1) S+1, \ldots, T \cdot S .
\end{aligned}
$$

The end of the last lot in some previous macro-period $t$ must leave sufficient time for the setup time $\omega_{l t+1}$ (11). The same time slot control enables us to constrain that the finishing time of one slot, minus the finishing time of the previous slot, must be at least as large as the production time in the corresponding slot, plus the required setup time in advance (12)-(13). Figure 6 illustrates the situation described by these constraints with $S=2$ and $s=s 1, s 2, s 3, s 4 ; \tau=1,2, \ldots, 10$; $t=1,2 ; C^{m}=1$ and $C=5$.
There is only one possible beginning and end for each slot in the time horizon (14)-(15). While $x_{j l s}$ indicates whether or not a certain slot is reserved to produce a particular product, $u_{l s}$ indicates whether or not that slot is indeed used to schedule a lot. If the slot is not used, then the lot size must be zero (16); otherwise, at least a small amount $\varepsilon$ must be produced (17). Time cannot be allocated for slots not effectively used $\left(u_{l s}=0\right)$, so $x_{l s \tau}^{B}=x_{l s \tau}^{E}$ must occur for the same micro-period $\tau$ (18)-(19). To avoid redundancy in the model, whenever a slot is not used, it should start as soon as the previous slot ends (20)-(21). The possibly empty slots in a macro-period will be the last ones in that macro-period (22). The start and end of a slot are chosen in such a way that the start indicates the first micro-period in which the lot is produced, if there is a real lot for that slot, and the end indicates the last microperiod (23)-(24). Figure 7 illustrates the situation described by these two constraints.

The term $\varepsilon \cdot u_{l s}$ in constraint (23) and the term $\sum_{j=1}^{J} p_{j l} q_{j l s}-C^{m}$ in constraint (24) only matter for the case when the first and last micro-periods of a slot are the same. Notice that the processing time of $P 3$ in Figure 7 is smaller than the micro-period capacity in constraint (24). However, constraint (23) ensures that a positive amount will be produced because we must have $u_{L 1, P 3}=1$ by constraint (16).

3.5. Production Line: Synchronization Constraints. The model must describe how the raw materials in tanks are handled by production lines

$$
q_{j l s}=\sum_{k=1}^{\bar{L}} \sum_{\tau=(t-1) T^{m}+1}^{t \cdot T^{m}} q_{k j l s \tau}
$$

$l=1, \ldots, L ; t=1, \ldots, T ; s=(t-1) S+1, \ldots, T \cdot S, \tau=$ $(t-1) T^{m}+1, \ldots, t \cdot T^{m}$,

$$
\begin{aligned}
\delta_{l s}= & \left(\sum_{\tau=(t-1) T^{m}+1}^{t \cdot T^{m}} \tau x_{l s \tau}^{E}-\sum_{\tau=(t-1) T^{m}+1}^{t \cdot T^{m}} \tau x_{l, s-1, \tau}^{B}+1\right) C^{m} \\
& -\sum_{j=1}^{J} p_{j l} q_{j l s},
\end{aligned}
$$




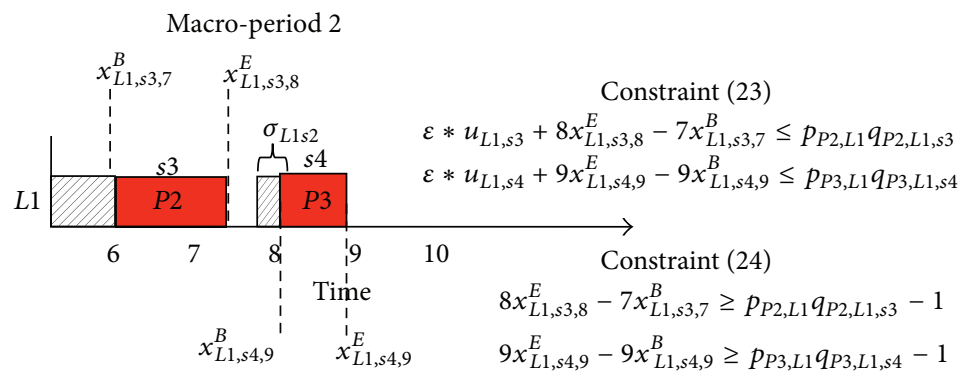

FIgURE 7: Time window for production.

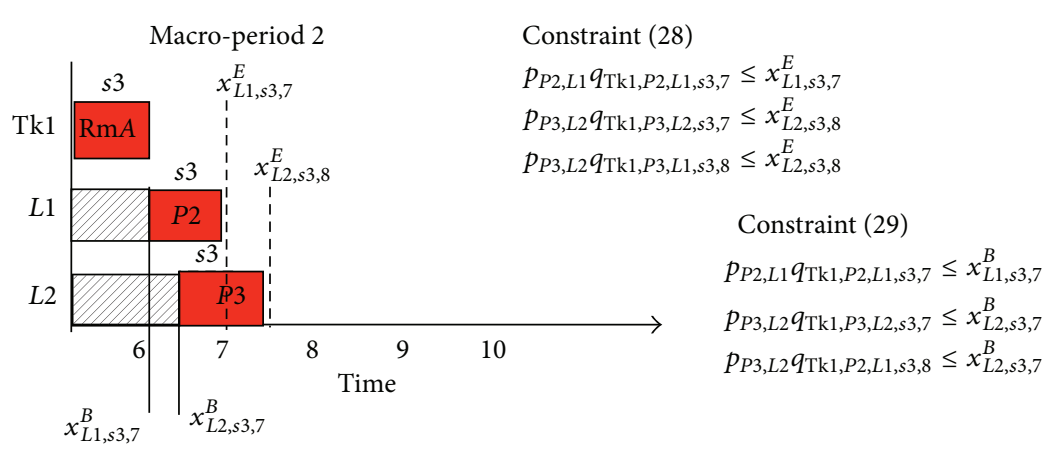

FIGURE 8: Synchronization of line productions with tank storage.

$l=1, \ldots, L ; t=1, \ldots, T ; s=(t-1) S+2, \ldots, T \cdot S, \tau=$ $(t-1) T^{m}+1, \ldots, t \cdot T^{m}$,

$$
\sum_{k=1}^{\bar{L}} \sum_{j=1}^{J} p_{j l} q_{k j l s \tau} \leq C^{m}-\delta_{l s}+\left(1-x_{l s \tau}^{B}\right) C^{m}
$$

$l=1, \ldots, L ; t=1, \ldots, T ; s=(t-1) S+1, \ldots, T \cdot S, \tau=$ $(t-1) T^{m}+1, \ldots, t \cdot T^{m}$,

$$
\sum_{k=1}^{\bar{L}} \sum_{j=1}^{J} p_{j l} q_{k j l s \tau} \leq \sum_{\tau^{\prime}=(t-1) T^{m}+1}^{t \cdot T^{m}} C^{m} x_{l s \tau^{\prime}}^{E},
$$

$l=1, \ldots, L ; t=1, \ldots, T ; s=(t-1) S+1, \ldots, T \cdot S, \tau=$ $(t-1) T^{m}+1, \ldots, t \cdot T^{m}$,

$$
\sum_{k=1}^{\bar{L}} \sum_{j=1}^{J} p_{j l} q_{k j l s \tau} \leq \sum_{\tau^{\prime}=(t-1) T^{m}+1}^{t \cdot T^{m}} C^{m} x_{l s \tau^{\prime}}^{B},
$$

$l=1, \ldots, L ; t=1, \ldots, T ; s=(t-1) S+1, \ldots, T \cdot S, \tau=$ $(t-1) T^{m}+1, \ldots, t \cdot T^{m}$.

To link production lines and tanks, we figure out which amount of soft drink being bottled on a line comes from which tank (25). In the first micro-period of a lot, sometimes $\delta_{l s}$ are reserved for setup or idle time, while the remaining time is production time (26) and (27). Of course, the soft drink taken from a tank must be taken during the time window in which the soft drink is filled into bottles (28)-(29). Figure 8 illustrates the situation described by these constraints with $C^{m}=1$. Let us suppose that $\operatorname{Rm} A$ is used to produce $P 2$ and $P 3$.

Notice that the processing time of $P 3$ takes from $\tau=7$ to $\tau=8$. This is considered by constraints (28) and (29).
3.6. Tanks: Usual Constraints. Now let us focus on usual lot sizing constraints for the tank level

$$
\begin{aligned}
& \bar{x}_{j k s}=0 \\
& j=1, \ldots, \bar{J} ; k \in\{1, \ldots, \bar{L}\} \backslash \bar{L}_{j} ; s=1, \ldots, T \cdot \bar{S}, \\
& \sum_{j=1}^{J} \bar{x}_{j k s}=1 \quad k=1, \ldots, \bar{L} ; s=1, \ldots, T \cdot \bar{S} \\
& \bar{q}_{j k s} \leq \bar{Q}_{k} \bar{x}_{j k s} \\
& j=1, \ldots, \bar{J}, k=1, \ldots, \bar{L} ; s=1, \ldots, T \cdot \bar{S}, \\
& \bar{q}_{j k s} \leq \bar{Q}_{k} \bar{u}_{k s} \\
& j=1, \ldots, \bar{J}, k=1, \ldots, \bar{L} ; s=1, \ldots, T \cdot \bar{S}, \\
& \sum_{j=1}^{\bar{J}} \bar{q}_{j k s} \geq \underline{Q}_{k} \bar{u}_{k s} \\
& j=1, \ldots, \bar{J}, k=1, \ldots, \bar{L} ; s=1, \ldots, T \cdot \bar{S}, \\
& \bar{z}_{i j k s} \geq \bar{x}_{j k s}+\bar{x}_{i k, s-1}-2+\bar{u}_{k s} \\
& i, j=1, \ldots, \bar{J}, k=1, \ldots, \bar{L} ; s=1, \ldots, T \cdot \bar{S}, \\
& \bar{x}_{j k s}-\bar{x}_{j k, s-1} \leq \bar{u}_{k s} \\
& j=1, \ldots, \bar{J}, k=1, \ldots, \bar{L} ; s=1, \ldots, T \cdot \bar{S},
\end{aligned}
$$




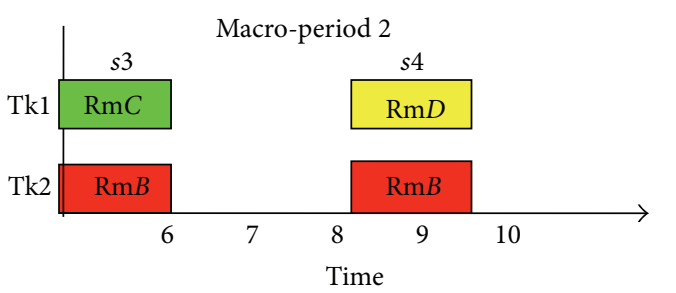

Constraint (35)

$\bar{z}_{\mathrm{RmC}, \mathrm{Rm} D, \mathrm{Tk} 1, s 4} \geq \bar{x}_{\mathrm{Rm} D, \mathrm{Tk} 1, s 4}+\bar{x}_{\mathrm{RmC}, \mathrm{Tk} 1, s 3}-2+\bar{u}_{\mathrm{Tk} 1, s 4}$

$\bar{z}_{\mathrm{RmC}, \mathrm{Rm} D, \mathrm{Tk} 1, s 4} \geq 1+1-2+1$

Constraint (36)

$$
\begin{aligned}
& \bar{x}_{\mathrm{Rm} D, \mathrm{Tk} 1, s 4}-\bar{x}_{\mathrm{Rm} D, \mathrm{Tk} 1, s 3} \leq \bar{u}_{\mathrm{Tk} 1, s 4} \Longrightarrow 1-0 \leq 1 \\
& \bar{x}_{\mathrm{RmC}, \mathrm{Tk} 1, s 4}-\bar{x}_{\mathrm{RmC}, \mathrm{Tk} 1, s 3} \leq \bar{u}_{\mathrm{Tk} 1, s 4} \Longrightarrow 0-1 \leq 1
\end{aligned}
$$

From constraints (31)-(34)

$\bar{x}_{\mathrm{Rm} B, \mathrm{Tk} 2, s 4}=\bar{x}_{\mathrm{Rm} B, \mathrm{Tk} 2, s 3}=\bar{u}_{\mathrm{Tk} 2, \mathrm{~s} 3}=1$

$\bar{x}_{\mathrm{Rm} D, \mathrm{Tk} 1, s 4}=\bar{x}_{\mathrm{RmD}, \mathrm{Tk} 1, s 3}=\bar{u}_{\mathrm{Tk} 1, s 4}=1$

$\bar{z}_{\mathrm{Rm} B, \mathrm{Rm} B, \mathrm{Tk} 2, s 4} \geq \bar{x}_{\mathrm{Rm} B, \mathrm{Tk} 2, s 4}+\bar{x}_{\mathrm{Rm} B, \mathrm{Tk} 2, \mathrm{~s} 3}-2+\bar{u}_{\mathrm{Tk} 2, s 4}$

$\bar{z}_{\mathrm{Rm} B, \operatorname{Rm} B, \mathrm{Tk} 2, s 4} \geq 1+1-2+1$

$\bar{x}_{\mathrm{Rm} B, \mathrm{Tk} 2, s 4}-\bar{x}_{\mathrm{Rm} B, \mathrm{Tk} 2, s 3} \leq \bar{u}_{\mathrm{Tk} 2, s 4} \Longrightarrow 1-1 \leq 1$

Figure 9: Raw material changeover.

$$
\begin{aligned}
\bar{\sigma}_{k s} & =\sum_{i=1}^{\bar{J}} \sum_{j=1}^{\bar{J}} \bar{\sigma}_{i j k}^{\prime} \bar{z}_{i j k s} \\
i, j & =1, \ldots, \bar{J}, k=1, \ldots, \bar{L} ; s=1, \ldots, T \cdot \bar{S} \\
\bar{\omega}_{k t s} & \leq \bar{\sigma}_{k,(t-1) S+1} \\
i, j & =1, \ldots, \bar{J}, k=1, \ldots, \bar{L} ; s=1, \ldots, T \cdot \bar{S} \\
\sum_{t=(t-1) S+1} \quad \bar{\sigma}_{k s} \leq C+\omega_{k t} & \\
i, j & =1, \ldots, \bar{J}, k=1, \ldots, \bar{L} ; s=1, \ldots, T \cdot \bar{S} .
\end{aligned}
$$

A particular raw material can be stored in some tanks (30) and tanks can store one raw material at a time (31). The tanks have a maximum capacity (32) and nothing can be stored, whenever the tank slot is not effectively used (33). However, a minimum lot size must be stored if a tank slot is used for some raw material (34). The setup state is not preserved in the tanks, that is, a tank must be setup again (cleaned and filled), even if the same soft drink has been in the tank just before. Care must be taken when setups occur in the model only if the tank is really used, because changing the reservation for a tank does not necessarily mean that a setup takes place (35)(36). Figure 9 illustrates these constraints.

From the schedule in Figure 9, constraints (31)-(34) define $\bar{x}_{\mathrm{RmB}, \mathrm{Tk} 2, s 4}=\bar{x}_{\mathrm{RmB}, \mathrm{Tk} 2, \mathrm{~s} 3}=\bar{u}_{\mathrm{Tk} 2, \mathrm{~s} 3}=1$ and $\bar{x}_{\mathrm{RmD}, \mathrm{Tk} 1, s 4}=\bar{x}_{\mathrm{RmD}, \mathrm{Tk} 1, s 3}=\bar{u}_{\mathrm{Tk} 1, s 4}=1$. Moreover, the raw materials scheduled will satisfy constraints (35)-(36). As in the production lines, it is also possible to determine the tank slot setup time (37) and the setup time that may be at the end of the previous macro-period (38)-(39). Thus, the setup time in front of lot $s$, at the beginning of some period, is the setup time value minus the fraction of setup time spent in the previous periods. This value cannot exceed the macro-period capacity $C$.

3.7. Tanks: Time Slot Constraints. The main point here is to keep control when the tank slots are used throughout the time horizon by production lines. This will help to know when a setup time in tanks will interfere with some production line or from which tanks the lines are taking the raw materials:

$$
\begin{aligned}
& \bar{q}_{j k s}=\sum_{\tau=(t-1) T^{m}+1}^{t \cdot T^{m}} \bar{q}_{j k s \tau}, \\
& j=1, \ldots, \bar{J}, k=1, \ldots, \bar{L} ; t=1, \ldots, T ; s=1, \ldots, T \cdot \bar{S}, \\
& t \cdot C-\sum_{\tau=(t-1) T^{m}+1}^{t \cdot T^{m}} C^{m} \tau \bar{x}_{k, t \cdot \bar{S}, \tau}^{E} \geq \omega_{k, t+1}, \\
& k=1, \ldots, \bar{L} ; t=1, \ldots, T-1 \text {; } \\
& \sum_{\tau=(t-1) T^{m}+1}^{t \cdot T^{m}} C^{m} \tau \bar{x}_{k s \tau}^{E}-\sum_{\tau=(t-1) T^{m}+1}^{t \cdot T^{m}} C^{m} \tau \bar{x}_{k, s-1, \tau}^{E} \geq \bar{\sigma}_{k s}, \\
& k=1, \ldots, \bar{L} ; t=1, \ldots, T ; s=(t-1) \bar{S}+1, \ldots, t \cdot \bar{S}, \\
& \sum_{\tau=(t-1) T^{m}+1}^{t \cdot T^{m}} C^{m} \tau \bar{x}_{k,(t-1) \bar{S}+1, \tau}^{E} \\
& \geq(t-1) C+\bar{\sigma}_{k,(t-1) \cdot \bar{S}+1}-\omega_{k t}, \\
& k=1, \ldots, \bar{L} ; t=1, \ldots, T \text {, } \\
& \sum_{\tau=(t-1) T^{m}+1}^{t \cdot T^{m}} \bar{x}_{k s \tau}^{E}=1
\end{aligned}
$$

$k=1, \ldots, \bar{L} ; t=1, \ldots, T ; s=(t-1) \bar{S}+1, \ldots, t \cdot \bar{S} ; \tau=$ $(t-1) T^{m}+1, \ldots, t \cdot T^{m}$,

$$
\sum_{\tau=(t-1) T^{m}+1}^{t \cdot T^{m}} \bar{x}_{k s \tau}^{B}=1,
$$

$k=1, \ldots, \bar{L} ; t=1, \ldots, T ; s=(t-1) \bar{S}+2, \ldots, t \cdot \bar{S}$

$$
\sum_{\tau=(t-2) T^{m}+1}^{t \cdot T^{m}} \bar{x}_{k,(t-1) \bar{S}+1, \tau}^{B}=1,
$$

$k=1, \ldots, \bar{L} ; t=2, \ldots, T$, 


$$
\sum_{\tau=1}^{T^{m}} \bar{x}_{k 1 \tau}^{B}=1
$$

$$
\begin{aligned}
& k=1, \ldots, \bar{L} ; t=2, \ldots, T \\
& \sum_{j=1}^{\bar{J}} \bar{q}_{j k s \tau} \leq \bar{Q}_{k} \bar{x}_{k s \tau}^{E},
\end{aligned}
$$

$k=1, \ldots, \bar{L} ; t=1, \ldots, T ; s=(t-1) \bar{S}+1, \ldots, t \cdot \bar{S} ; \tau=$ $(t-1) T^{m}+1, \ldots, t \cdot T^{m}$,

$$
\begin{gathered}
\sum_{\tau=(t-1) T^{m}+1}^{t \cdot T^{m}} \tau \bar{x}_{k s \tau}^{E}-\sum_{\tau=(t-1) T^{m}+1}^{t \cdot T^{m}} \tau \bar{x}_{k s \tau}^{B} \leq T^{m} \bar{u}_{k s}, \\
k=1, \ldots, \bar{L} ; t=1, \ldots, T ; s=(t-1) \bar{S}+2, \ldots, t \cdot \bar{S} ; \\
\sum_{\tau=(t-1) T^{m}+1}^{t \cdot T^{m}} \tau \bar{x}_{k,(t-1) \bar{S}+1, \tau}^{E}-\sum_{\tau=(t-2) T^{m}+1}^{t \cdot T^{m}} \tau \bar{x}_{k,(t-1) \bar{S}+1, \tau}^{B} \\
\leq T^{m} \bar{u}_{k,(t-1) \bar{S}+1}, \quad \\
k=1, \ldots, \bar{L} ; t=2, \ldots, T ; \\
\sum_{\tau=1}^{T^{m}} \tau \bar{x}_{k 1 \tau}^{E}-\sum_{\tau=1}^{T^{m}} \tau \bar{x}_{k 1 \tau}^{B} \leq T^{m} \bar{u}_{k 1},
\end{gathered}
$$$$
k=1, \ldots, \bar{L},
$$$$
\sum_{\tau=(t-1) T^{m}+1}^{t \cdot T^{m}} \tau \bar{x}_{k s \tau}^{B}-\sum_{\tau=(t-1) T^{m}+1}^{t \cdot T^{m}} \tau \bar{x}_{k s \tau}^{E} \leq \bar{u}_{k s},
$$$$
k=1, \ldots, \bar{L} ; t=1, \ldots, T ; s=(t-1) \bar{S}+2, \ldots, t \cdot \bar{S}
$$$$
\sum_{\tau=(t-2) T^{m}+1}^{t \cdot T^{m}} \tau \bar{x}_{k,(t-1) \bar{S}+1, \tau}^{B}-\sum_{\tau=(t-1) T^{m}+1}^{t \cdot T^{m}} \tau \bar{x}_{k,(t-1) \bar{S}+1, \tau}^{E}
$$$$
\leq \bar{u}_{k,(t-1) \bar{S}+1},
$$$$
k=1, \ldots, \bar{L} ; t=2, \ldots, T
$$$$
\sum_{\tau=1}^{T^{m}} \tau \bar{x}_{k 1 \tau}^{B}-\sum_{\tau=1}^{T^{m}} \tau \bar{x}_{k 1 \tau}^{E} \leq \bar{u}_{k 1}
$$

$k=1, \ldots, \bar{L}$

$$
\sum_{\tau=(t-1) T^{m}+1}^{t \cdot T^{m}} \tau \bar{x}_{k s \tau}^{B}-\sum_{\tau=(t-1) T^{m}+1}^{t \cdot T^{m}} \tau \bar{x}_{k, s-1, \tau}^{E} \leq T^{m} \bar{u}_{k s},
$$

$k=1, \ldots, \bar{L} ; t=1, \ldots, T ; s=(t-1) \bar{S}+2, \ldots, t \cdot \bar{S}$

$$
\begin{aligned}
& \sum_{\tau=(t-1) T^{m}+1}^{t \cdot T^{m}} \tau \bar{x}_{k,(t-1) \bar{S}+1, \tau}^{B}-\left((t-1) T^{m}+1\right) \\
& \quad \leq T^{m} \bar{u}_{k,(t-1) \bar{S}+1},
\end{aligned}
$$

$k=1, \ldots, \bar{L} ; t=2, \ldots, T$

$$
\sum_{\tau=1}^{T^{m}} \tau \bar{x}_{k 1 \tau}^{B}-1 \leq T^{m} \bar{u}_{k 1}
$$

$k=1, \ldots, \bar{L}$

$$
\bar{u}_{k s} \geq \bar{u}_{k, s+1}
$$

$$
k=1, \ldots, \bar{L} ; t=1, \ldots, T ; s=(t-1) \bar{S}+1, \ldots, t \cdot \bar{S}-1
$$$$
C^{m} \bar{u}_{k s}+\sum_{\tau=(t-1) T^{m}+1}^{t \cdot T^{m}} C^{m} \bar{\tau} x_{k s \tau}^{E}-\sum_{\tau=(t-1) T^{m}+1}^{t \cdot T^{m}} C^{m} \bar{\tau} x_{k s \tau}^{B}
$$$$
=\bar{\sigma}_{k s}
$$

$k=1, \ldots, \bar{L} ; t=1, \ldots, T ; s=(t-1) \bar{S}+2, \ldots, t \cdot \bar{S}$

$$
C^{m} \bar{u}_{k,(t-1) \bar{S}+1}+\sum_{\tau=(t-1) T^{m}+1}^{t \cdot T^{m}} C^{m} \bar{\tau} x_{k,(t-1) \bar{S}+1, \tau}^{E}
$$

$$
-\sum_{\tau=(t-2) T^{m}+1}^{t \cdot T^{m}} C^{m} \bar{\tau} x_{k,(t-1) \bar{S}+1, \tau}^{B}=\bar{\sigma}_{k,(t-1) \bar{S}+1},
$$

$$
\begin{aligned}
& k=1, \ldots, \bar{L} ; t=2, \ldots, T \\
& \quad C^{m} \bar{u}_{k 1}+\sum_{\tau=1}^{T^{m}} C^{m} \bar{\tau} x_{k 1 \tau}^{E}-\sum_{\tau=1}^{T^{m}} C^{m} \bar{\tau} x_{k 1 \tau}^{B}=\bar{\sigma}_{k 1}-\bar{\omega}_{k 1},
\end{aligned}
$$

$k=1, \ldots, \bar{L}$

$$
\sum_{j=1}^{\bar{J}} \bar{I}_{j k, \tau-1} \leq \bar{Q}_{k}\left(\left(1-\bar{x}_{k s \tau}^{B}\right)+\left(1-\bar{u}_{k s}\right)\right),
$$

$k=1, \ldots, \bar{L} ; t=1, \ldots, T-1 ; s=(t-1) \bar{S}+1, \ldots, t \cdot \bar{S}+1$; $\tau=(t-1) T^{m}+1, \ldots, t \cdot T^{m}$

$$
\sum_{j=1}^{\bar{J}} \bar{I}_{j k, \tau-1} \leq \bar{Q}_{k}\left(\left(1-\bar{x}_{k s \tau}^{B}\right)+\left(1-\bar{u}_{k s}\right)\right),
$$

$k=1, \ldots, \bar{L} ; s=(T-1) \bar{S}+1, \ldots, T \cdot \bar{S}+1 ; \tau=(T-1) T^{m}+$ $1, \ldots, T \cdot T^{m}$.

Most of these constraints are similar to those presented for the production line level. The amount that is filled into a tank must be scheduled, so that the micro-period in which the raw material comes into the tank is known (40). Tank slots must be scheduled in such a way that the setup time that corresponds to a slot fits into the time window between the end of the previous slot and the end of the slot under consideration (41)-(42). The setup for the first slot in a macro-period may overlap the macro-period border to the previous macroperiod (43). This is the same idea presented by Figure 6 for lines. The finishing time of a scheduled slot must be unique, as must its starting time (44)-(47). The raw material is available for bottling just when the tank setup is done (48). Therefore, we must have $\bar{x}_{\mathrm{Tk} 2, s 3,7}^{E}=\bar{x}_{\mathrm{Tk} 2, s 4,9}^{E}=1$ in constraint (48) for the example shown in Figure 10. 


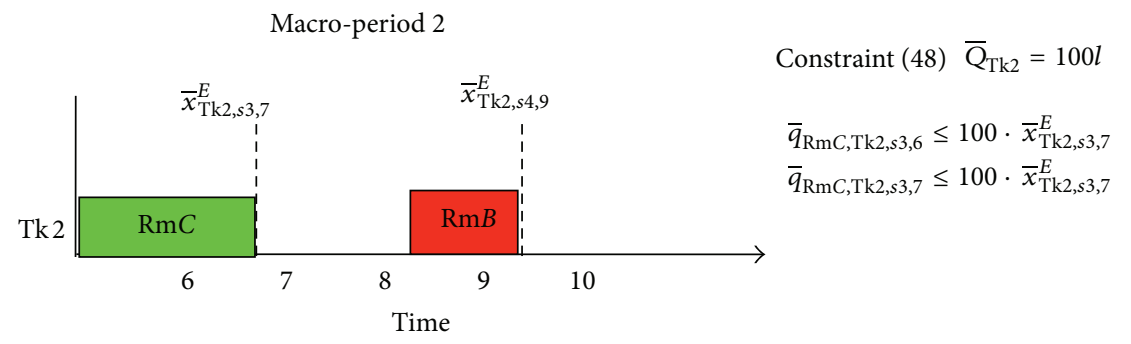

FIGURE 10: Tank refill before the end of the setup time.

The time window in which a slot is scheduled must be positive if and only if that slot is used to set up the tank (49)-(54). Redundancy can be avoided if empty slots are scheduled right after the end of previous slots (55)-(57). More redundancy can be avoided if we enforce that, within a macro-period, the unused slots are the last ones (58).

Without loss of generality, we can assume that the time needed to set up a tank is an integer multiple of the microperiod. Hence, the beginning and end of a slot must be scheduled so that this window equals exactly the time needed to set the tank up (59)-(61). Constraint (61) adds up microperiods $\tau=1, \ldots, T^{m}$ seeking the beginning and end of the tank setup in slot $\bar{s}=1$. Finally, a tank can only be set up again if it is empty (62)-(63). Notice that a tank slot reservation $\left(\bar{x}_{k s \tau}^{B}=1\right)$ does not mean that the tank should be previously empty $\left(\bar{I}_{j k \tau-1}=0\right)$. The tank slot also has to be effectively used $\left(\bar{u}_{k s}=1\right.$ and $\left.\bar{x}_{k s \tau}^{B}=1\right)$ in (62)-(63). Constraint (63) describes this situation for the last macro-period $T$ where the first slot of the next macro-period is not taken into account.

Finally, notice that there are group of equations, as for example, (45)-(47), (49)-(51), (52)-(57), (55)-(57), and (59)(61), that are derived from the same equations, respectively, (45), (49), (52), (55), and (59). These derived equations are necessary to deal with the first slot and sometimes with the last slot in each macro-period.

3.8. Tanks: Synchronization Constraints. The model must describe how the products in production lines are handled by tanks:

$$
\begin{aligned}
& \bar{I}_{j k \tau}=\bar{I}_{j k, \tau-1}+\sum_{s=(t-1) S+1}^{t \cdot S} \bar{q}_{j k s \tau} \\
& -\sum_{i=1}^{J} \sum_{l=1}^{L} \sum_{s=(t-1) S+1}^{t \cdot S} r_{j i} q_{k i l s \tau}, \\
& \bar{I}_{j k \tau} \geq \sum_{i=1}^{J} \sum_{l=1}^{L} \sum_{s=(t-1) S+1}^{t \cdot S} r_{j i} q_{k i l s, \tau+1},
\end{aligned}
$$

What is in the tank at the end of a micro-period must be what was in the tank at the beginning of that micro-period,

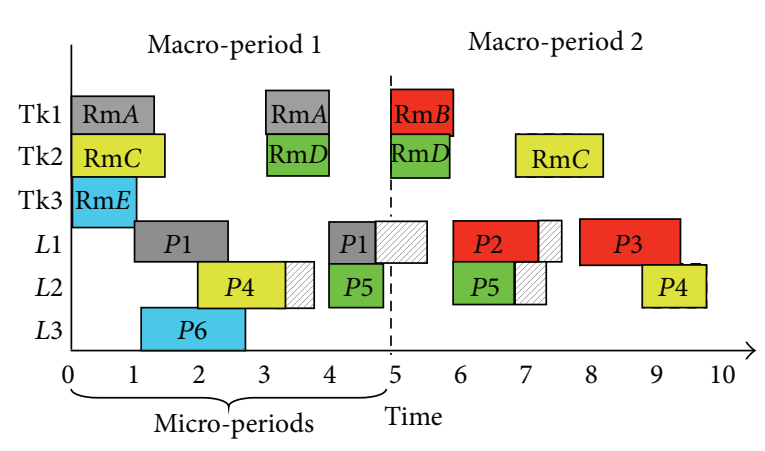

Figure 11: Possible solution for SITLSP.

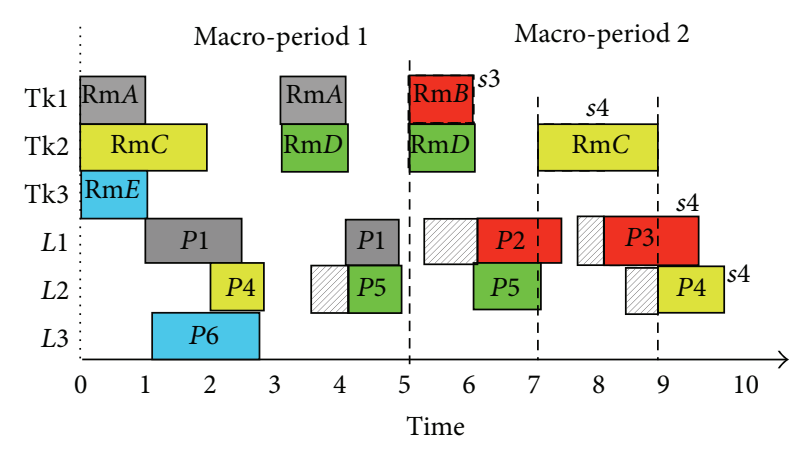

FIGURE 12: Possible solution provided by the model.

plus what comes into the tank in that micro-period, minus what is taken from that tank for being bottled in production lines (64). To coordinate the production lines and the tanks, it is required that what is taken out of a tank must have been filled into the tank at least one micro-period ahead (65).

3.9. Example. This subsection will present an example to illustrate what kind of information is returned by a model solution. Let us start by the solution illustrated in Figure 5. This solution is shown again in Figure 11. Figure 12 presents the same solution considering the model assumptions.

According to the model, the setup times of the tanks are considered as an integer multiple of the time of a microperiod. For example, the setup time of $\mathrm{RmC}$ in Figure 11 goes from the entire $\tau=1$ (micro-period 1) until part of $\tau=2$ and from the entire $\tau=8$ until part of $\tau=9$. These setup times fit into two complete micro-periods in Figure 12 (see constraints (59)-(61)). The same happens with the setup time of $\mathrm{Rm} B$ 
TABLE 2: Parameter values.

\begin{tabular}{lccccccc}
\hline Param. & Values & Param. & Values & Param. & Values & Param. & Values \\
\hline$L$ & $2 ; 3 ; 4$ & $\bar{L}$ & $2 ; 3$ & $h_{j}$ & $1(\$ / \mathrm{u})$ & $\bar{h}_{j}$ & $1(\$ / \mathrm{u})$ \\
$J$ & $2 ; 3 ; 4$ & $\bar{J}$ & $1 ; 2$ & $\bar{h}_{j}$ & $1(\$ / \mathrm{u})$ & $\bar{h}_{j}$ & $1(\$ / \mathrm{u})$ \\
$S$ & $2 ; 3$ & $\bar{S}$ & 2 & $v_{j l}$ & $1(\$ / \mathrm{u})$ & $\bar{v}_{j k}$ & $1(\$ / \mathrm{u})$ \\
$T$ & $1 ; 2 ; 3 ; 4$ & $T^{m}$ & 5 & $Q_{k}$ & 10001 & $\bar{Q}_{k}$ & 50001 \\
$C$ & 5 t.u. & $C^{m}$ & 1 t.u. & min Dem & $500 \mathrm{u}$ & $\max D e m$ & $10000 \mathrm{u}$ \\
\hline
\end{tabular}

TABle 3: Parameter combination for each set of instances.

\begin{tabular}{lccccc}
\hline Comb. & $L / \bar{L} / J / \bar{J}$ & Comb. & $L / \bar{L} / J / \bar{J}$ & Comb. & $L / \bar{L} / J / \bar{J}$ \\
\hline$S 1$ & $2 / 2 / 2 / 1$ & $S 4$ & $3 / 3 / 2 / 1$ & $S 7$ & $4 / 3 / 2 / 1$ \\
$S 2$ & $2 / 2 / 3 / 2$ & $S 5$ & $3 / 3 / 3 / 2$ & $S 8$ & $4 / 3 / 3 / 2$ \\
$S 3$ & $2 / 2 / 4 / 2$ & $S 6$ & $3 / 3 / 4 / 2$ & $S 9$ & $4 / 3 / 4 / 2$ \\
\hline
\end{tabular}

(macro-period 2). The setup time of products in lines $L 1$ and $L 2$ are positioned in front of the next slot as required by constraints (8)-(13), and all the productions in the lines start after the tank setup time is finished (constraints (25), (28)(29), (40), and (48)).

The satisfaction of the model constraints imposes that binary variables $x_{j l s}, \bar{x}_{j k s}, u_{l s}, \bar{u}_{k s}, x_{l s \tau}^{B}, x_{l s \tau}^{E}, \bar{x}_{k s \tau}^{B}$, and $\bar{x}_{k s \tau}^{E}$ must be necessarily active (value 1 ) for lines, tanks, products, and raw materials in the slots and micro-periods shown in Figure 12. These active variables provide enough information about what and when the products and raw materials are scheduled throughout the time horizon. Moreover, the continuous variables $q_{j l s}, q_{k j l s \tau} \bar{q}_{j k s \tau}, \bar{q}_{j k s}$ must be positive for lines, tanks, products, and raw materials in the same slots and micro-periods.

For example, based on Figure 12, the model solution has positive variables $\bar{q}_{\mathrm{RmC}, \mathrm{Tk} 2,54,8}$ and $\bar{q}_{\mathrm{RmC}, \mathrm{Tk} 2, s 4,9}$ and returns the amount of $\mathrm{RmC}$ filled in Tk2 during micro-periods $\tau=$ 8 and $\tau=9$. The total filled in this slot $(s 4)$ is provided by $\bar{q}_{\mathrm{RmC}, \mathrm{Tk} 2, s 4}$. The model variable $q_{\mathrm{Tk} 2, P 4, L 2,10}$ returns the amount used by $P 4$ in $L 2$ which is taken from $\bar{q}_{\mathrm{RmC}, \mathrm{Tk} 2, \mathrm{~s} 4}$. In the same way, $q_{\mathrm{Tk} 1, P 3, L 1, s 4,9}$ and $q_{\mathrm{Tk} 1, P 3, L 1, s 4,10}$ have the lot sizes of $P 3$ taken from slot $s 3$ in variable $\bar{q}_{\mathrm{RmB}, \mathrm{Tk} 1, s 3}$. In this case, the total lot size $P 3$ is provided by $q_{P 3, L 1, s 4}$. All these variables returned by the model will provide enough information to build an integrated and synchronized schedule for lines and tanks.

\section{Computational Results for Small-to-Moderate Size Instances}

The main objectives in this section are to provide a set of benchmark results for the problem and to give an idea about the complexity of the model resolution. The lack of similar models in the literature has led us to create a set of instances for the SITLSP. An exact method available in an optimization package was used as the first approach to solve these instances. Table 2 shows the parameter values adopted.

A combination of parameters $L, \bar{L}, J$, and $\bar{J}$ defines a set of instances. These instances are considered of smallto-moderate sizes if compared to the industrial instances found in soft drink companies. Table 3 presents the parameter values that define each combination $S 1-S 9$. There are 9 possible combinations for macro-period $T=1,2,3$, and 4. For each one of these sets, 10 replications are randomly generated, resulting in $4 \times 9 \times 10=360$ instances in total. The other parameters of the model are randomly generated from a uniform distribution in the interval $[a, b]$, as shown in Table 4. Most of these parameters were defined following suggestions provided by the decision maker of a real-world soft drink company. The setup costs are proportional to setup times with parameter $f$ set to 1000 . This provides a suitable tradeoff between the different terms of the objective function.

The computational tests ran in a core 2 Duo, $2.66 \mathrm{GHz}$, and 1.95 GB RAM. The instances were coded using the modeling language AMPL and solved by the branch \& cut exact algorithm available in the solver CPLEX 11.0. The optimization package AMPL/CPLEX ran over each instance once during the time limit of one hour. Two kinds of problem solutions were returned: the optimal solution or the best feasible solution achieved up to the time limit. The following gap value was used:

$$
\operatorname{Gap}(\%)=100\left(\frac{Z-\bar{Z}}{\bar{Z}}\right)
$$

where $Z$ is the solution value and $\bar{Z}$ is the lower bound value returned by AMPL/CPLEX. Table 5 presents the computational results for $T=1,2$. Column Opt. has the number of optimal solutions found in each combination.

Column Gap(\%) shows the average gap of the final solutions returned by AMPL/CPLEX from the lower bound found. The CPU values are the average time spent (in seconds) to return these solutions. Table 6 shows the model figures for each combination. In Table 5 notice that the AMPL/CPLEX had no major problems to find solutions for $T=1$ and $T=2$. The solver was able to optimally solve all instances for $T=1$ (90 in 90) and found several optimal solution for $T=2$ (72 in 90) within the time limit. The required average CPU running times increased from $T=1$ to $T=2$, which is expected, based on the number of constraints and variables of the model for each instance (see Table 6). 
TABLE 4: Parameter ranges.

\begin{tabular}{lcl}
\hline Par. & Ranges & Meaning \\
\hline$\sigma_{i j l}^{\prime}$ & $U[0.5 ; 1]$ & Setup time (hours) from product $i$ to $j$ in line $l$ \\
$\bar{\sigma}_{i j k}^{\prime}$ & $U[1 ; 2]$ & Setup time (hours) from raw material $i$ to $j$ in tank $k$ \\
$s_{i j l}^{\prime}$ & $s_{i j l}=f \cdot \sigma_{i j l}^{\prime}$ & Line setup cost \\
$\bar{s}_{i j k}$ & $\bar{s}_{i j k}=f \cdot \bar{\sigma}_{i j k}^{\prime}$ & Tank setup cost \\
$p_{j l}$ & $U[1000 ; 2000]$ & Processing time (units/hour) of product $i$ in line $l$ \\
$r_{j i}$ & $U[0.3 ; 3]$ & Liters of raw material $i$ used to produce one unit of product $j$ \\
\hline
\end{tabular}

TABLE 5: AMPL/CPLEX results for the set of instances with $T=1,2$.

\begin{tabular}{|c|c|c|c|c|c|c|}
\hline \multirow{2}{*}{ Comb. } & \multicolumn{3}{|c|}{$T=1$} & \multicolumn{3}{|c|}{$T=2$} \\
\hline & Opt. & Gap(\%) & CPU & Opt. & $\mathrm{Gap}(\%)$ & $\mathrm{CPU}$ \\
\hline$S 1$ & 10 & 0 & 0.22 & 10 & 0.51 & 1.90 \\
\hline$S 2$ & 10 & 0 & 0.48 & 9 & 0.85 & 585.87 \\
\hline$S 3$ & 10 & 0 & 0.60 & 8 & 0.61 & 1271.04 \\
\hline$S 4$ & 10 & 0 & 0.66 & 10 & 0.32 & 15.49 \\
\hline S5 & 10 & 0 & 1.46 & 8 & 1.52 & 1414.51 \\
\hline S6 & 10 & 0 & 12.30 & 6 & 3.77 & 1519.31 \\
\hline S7 & 10 & 0 & 0.96 & 10 & 0.08 & 525.46 \\
\hline$S 8$ & 10 & 0 & 4.37 & 6 & 0.79 & 2052.72 \\
\hline$S 9$ & 10 & 0 & 7.83 & 5 & 1.38 & 1821.96 \\
\hline
\end{tabular}

TABLE 6: Model figures for the set of instances with $T=1,2$.

\begin{tabular}{|c|c|c|c|c|c|c|}
\hline \multirow{3}{*}{ Comb. } & \multicolumn{3}{|c|}{$T=1$} & \multicolumn{3}{|c|}{$T=2$} \\
\hline & \multicolumn{2}{|c|}{ Var. } & \multirow{2}{*}{ Const. } & \multicolumn{2}{|c|}{ Var. } & \multirow{2}{*}{ Const. } \\
\hline & Bin. & Cont. & & Bin. & Cont. & \\
\hline$S 1$ & 100 & 263 & 281 & 210 & 533 & 575 \\
\hline$S 2$ & 136 & 499 & 454 & 282 & 1004 & 919 \\
\hline$S 3$ & 142 & 615 & 512 & 294 & 1235 & 1039 \\
\hline$S 4$ & 150 & 452 & 419 & 315 & 916 & 862 \\
\hline S5 & 204 & 880 & 673 & 423 & 1771 & 1368 \\
\hline S6 & 213 & 1098 & 764 & 441 & 2206 & 1552 \\
\hline S7 & 176 & 555 & 498 & 367 & 1122 & 1013 \\
\hline$S 8$ & 246 & 1100 & 821 & 507 & 2211 & 1641 \\
\hline$S 9$ & 258 & 1390 & 924 & 531 & 2790 & 1865 \\
\hline
\end{tabular}

The AMPL/CPLEX was also able to find optimal solutions for $T=3$ (43 in 90) and $T=4$ (26 in 90) within one hour, but this value decreased as shown in Table 7 when compared with those in Table 6 . The complexity of the model proposed here, caused by the high number of binary/continuous variables and constraints, as shown in Table 8, explains the hardness faced by the branch and cut algorithm to achieve optimal solutions. Nevertheless, around $64 \%$ of the smallto-moderate size instances were optimally solved with 129 nonoptimal (feasible) solutions returned. The average gap from lower bounds, Gap(\%), has increased following the complexity of each set of instances. However, gap values are below $7 \%$ in all set of instances.

These small-to-moderate size instances were already used by Toledo et al. [39] where the authors proposed a multipopulation genetic algorithm to solve the SITLSP. These instances were solved and these solutions were helpful to adjust and evaluate the evolutionary method performance. The insights provided by this evaluation were also useful to set the evolutionary method to solve real-world instances of the problem.

\section{Computational Results for Moderate-to-Large Size Instances}

The previous experiments with the AMPL/CPLEX have shown how difficult it can be to find proven optimal solutions for the SITLSP within one hour of execution time, even for small-to-moderate size instances. However, they also showed that it is possible to find reasonably good feasible solutions under this computational time. In practical settings, decision makers are usually more interested in obtaining approximate 
TABLE 7: AMPL/CPLEX results for the set of instances with $T=3,4$.

\begin{tabular}{lccccc}
\hline Comb. & Tpt. & $\begin{array}{c}T=3 \\
\text { Gap }(\%)\end{array}$ & CPU & Opt. & $\begin{array}{c}T=4 \\
\text { Gap }(\%)\end{array}$ \\
\hline$S 1$ & 10 & 0.72 & 10.64 & 9 & 0.13 \\
CPU
\end{tabular}

TABLE 8: Model figures for the set of instances with $T=3,4$.

\begin{tabular}{|c|c|c|c|c|c|c|}
\hline \multirow{2}{*}{ Comb. } & \multicolumn{3}{|c|}{$T=3$} & \multicolumn{3}{|c|}{$T=4$} \\
\hline & Bin. Var. & Cont. Var. & Const. & Bin. Var. & Cont. Var. & Const. \\
\hline$S 1$ & 320 & 803 & 863 & 430 & 1073 & 1163 \\
\hline$S 2$ & 574 & 2014 & 1835 & 574 & 2014 & 1827 \\
\hline$S 3$ & 598 & 2475 & 2075 & 598 & 2475 & 2075 \\
\hline$S 4$ & 480 & 1380 & 1303 & 645 & 1844 & 1752 \\
\hline S5 & 642 & 2662 & 2071 & 861 & 3553 & 2764 \\
\hline S6 & 669 & 3314 & 2350 & 897 & 4422 & 3140 \\
\hline$S 7$ & 558 & 1689 & 1536 & 749 & 2256 & 2039 \\
\hline$S 8$ & 768 & 3322 & 2466 & 1029 & 4433 & 3335 \\
\hline$S 9$ & 804 & 4190 & 2799 & 1077 & 5590 & 3735 \\
\hline
\end{tabular}

TABLE 9: Parameter combination for each set of instances.

\begin{tabular}{lccc}
\hline Comb. & $L / \bar{L} / J / \bar{J} / T$ & Comb. & $L / \bar{L} / J / \bar{J} / T$ \\
\hline$L 1$ & $5 / 5 / 10 / 5 / 4$ & $L 6$ & $5 / 6 / 15 / 8 / 8$ \\
$L 2$ & $5 / 6 / 15 / 8 / 4$ & $L 7$ & $8 / 5 / 10 / 5 / 8$ \\
$L 3$ & $8 / 5 / 10 / 5 / 4$ & $L 8$ & $8 / 6 / 15 / 8 / 8$ \\
$L 4$ & $8 / 6 / 15 / 8 / 4$ & $L 9$ & $5 / 5 / 10 / 5 / 12$ \\
$L 5$ & $5 / 5 / 10 / 5 / 8$ & $L 10$ & $5 / 6 / 15 / 8 / 12$ \\
\hline
\end{tabular}

solutions found in shorter times than optimal solutions which require much longer runtimes.

For this reason, instead of spending longer time looking for optimal solutions, this section evaluates some alternatives to find reasonably good approximate solutions for moderateto-large size instances. For this, another combination of parameters $L / \bar{L} / J / \bar{J} / T$ is considered, which defines a more complex and realistic set of instances, namely, $L 1-L 10$. Table 9 shows the corresponding parameter values.

While the model parameters were changed to $C=10 \mathrm{t}$.u, $T^{m}=10, S=5,8$, and $\bar{S}=3,4$, the remaining parameters kept the same values of the ones shown in Tables 2 and 4 . For each one of the 10 possible combinations in Table 9, a total of 10 replications was randomly generated resulting in 100 instances. Preliminary experiments with some of these instances showed that even feasible solutions are difficult to find after executing AMPL/CPLEX for one hour. Therefore, two relaxations of the model were investigated: the first
TABLE 10: Relaxation approaches results.

\begin{tabular}{lcccccc}
\hline Comb. & \multicolumn{3}{c}{$R 1$} & & \multicolumn{2}{c}{$R 2$} \\
& Opt. & Feas. & Penal & Opt. & Feas. & Penal \\
\hline$L 1$ & 10 & - & - & - & 10 & - \\
$L 2$ & 10 & - & - & - & 9 & 1 \\
$L 3$ & 9 & 1 & - & - & 9 & 1 \\
$L 4$ & 4 & 6 & - & - & 2 & 8 \\
$L 5$ & 8 & 2 & - & - & 8 & 2 \\
$L 6$ & 4 & 6 & - & - & 3 & 7 \\
$L 7$ & 6 & 4 & - & - & 3 & 7 \\
$L 8$ & 5 & 5 & - & - & - & 10 \\
$L 9$ & 7 & 3 & - & - & 7 & 3 \\
$L 10$ & - & 5 & 5 & - & 4 & 6 \\
\hline
\end{tabular}

relaxation, $R 1$, keeps as binary variable only the model variables $u_{l s}$ and $\bar{u}_{k s}$, whereas the other binary variables are set continuous in the range $[0,1]$. The second relaxation, $R 2$, keeps $u_{l s}$ and $\bar{u}_{k s}$ as binary as well as $x_{j l s}$ and $\bar{x}_{j k s}$. A similar idea was used by Gupta \& Magnusson [21] to deal with a onelevel capacitated lot sizing and scheduling problem.

These two relaxation approaches were applied to solve the set of instances $L 1-L 10$ within one hour. The results are shown in Table 10, where Opt. is the number of optimal solutions found by $R 1$ and $R 2$, respectively, Feas. is the number of feasible solutions without penalties, and Penal is the number of feasible solutions with penalties. Remember that 


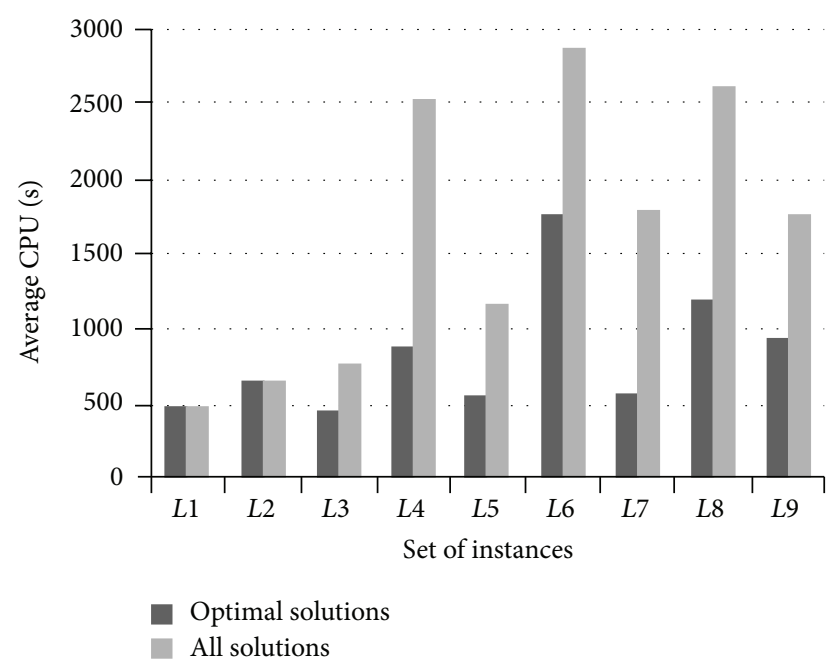

FIgURE 13: Average CPU time to find solutions.

nonsatisfied demands are penalized in the objective function of the model (see (1)).

Indeed for moderate-to-large size instances, approach $R 1$ succeeded in finding optimal solutions for a total of 63 out of 100 instances. Approach $R 2$ returned only feasible solutions with some of them penalized. A total of 55 out of 100 solutions without penalties were found by $R 2$.

Figure 13 shows the average CPU time spent by $R 1$ to solve 10 instances on each set $L 1-L 9$. This average time was calculated for the whole set of instances, as well as for only that subset of instances with optimal solutions found. All solutions were returned in less than $3000 \mathrm{sec}$. on average, and optimal solutions took less than $2000 \mathrm{sec}$. Thus, $R 1$ and $R 2$ approaches returned approximate solutions within a reasonable computational time for these sets of large size instances.

\section{Conclusions}

A great deal of scientific work has been done for decades to study lot sizing problems and combined lot sizing and scheduling problems, respectively. A whole bunch of stylized model formulations has been published under all kinds of assumptions to investigate this class of planning problems. It is well understood that certain aspects make planning become a hard task not only in theory. Among these issues are capacity constraints, setup times, sequence dependencies, and multilevel production processes, just to name a few. Many researchers including ourselves have focused on such stylized models and spent a lot of effort on solving artificially created instances with tailor-made procedures knowing that even small changes of the model would require the development of a new solution procedure. But very often, it remains unclear whether or not the stylized model represents a practical problem. Hence, we feel that it is necessary to move a bit more towards real applications and start to motivate the models being used stronger than before.

An attempt to follow this paradigm is presented in this paper. We were in contact with a company that bottles very well-known soft drinks and closely observed the main production process where different flavors are produced and filled into bottles of different sizes. The production planning problem is a lot sizing and scheduling problem with several thorny issues that have been discussed in the literature before, for example, capacity constraints, setup times, sequence dependencies, and multilevel production processes. But in addition to the issues described in existing literature, many specific aspects make this planning problem different from what we know from the literature. Hence, we contributed to the field by describing in detail what the practical problem is so that future researchers may use this problem instead of a stylized one to motivate their research.

Furthermore, we succeeded in putting together a model for the practical problem by making use of existing ideas from the literature, that is, we combined two stylized model formulations, the GLSP and the CSLP, to formulate a model for the practical situation. In this manner, this paper contributes to the field, because it proves a posteriori that the stylized models studied before are indeed relevant if used in a proper way which is not obvious. As the focus of this paper is not on sophisticated solution procedures, we used commercial software to solve small and medium sized instances to give the reader a practical grasp on the complexity of the problem. A next step could be to work on model reformulations given that nowadays commercial software is quite strong if provided with a strong model formulation. Future work may also focus on tailor-made algorithms, of course. We believe that heuristics are most appropriate for such real-world problems, but work on optimum solution procedures or lower bounds is relevant, too, because benchmark results will be needed.

\section{Appendix}

\section{Symbols for Parameters and Decision Variables}

\section{General Parameters}

$T$ : number of macro-periods,

$C$ : capacity (in time units) within a macro-period, $T^{m}$ : number of micro-periods per macro-period, $C^{m}$ : capacity (in time units) within a micro-period $\left(C=T^{m} \times C^{m}\right)$, $\varepsilon$ : a very small positive, real-valued number, $M$ : a very large number.

\section{Parameters for Linking Production Lines and Tanks}

$r_{j i}$ : amount of raw material $j$ to produce one unit of product $i$.

\section{Decision Variables for Linking Production Lines and Tanks}

$q_{k j l s t}$ : amount of product $j$, which is produced in line $l$ in micro-period $\tau$ and which belongs to lot $s$ that uses raw material from tank $k$. 


\section{Parameters for the Production Lines}

$J$ : number of products,

$L:$ number of parallel lines,

$L_{j}$ : set of lines on which product $j$ can be produced,

$S$ : upper bound on the number of lots per macroperiod, that is, the number of slots in production lines,

$d_{j t}$ : demand for product $j$ at the end of macro-period $t$,

$s_{i j l}$ : sequence-dependent setup cost coefficient for a setup from product $i$ to $j$ in line $l\left(s_{j j l}=0\right)$,

$h_{j}$ : holding cost coefficient for having one unit of product $j$ in inventory at the end of a macro-period,

$v_{j l}$ : production cost coefficient for producing one unit of product $j$ in line $l$,

$p_{j l}$ : production time needed to produce one unit of product $j$ in line $l$ (assumption: $p_{j l} \leq C^{m}$ ),

$x_{j l 0}=1$, if line $l$ is set up for product $j$ at the beginning of the planning horizon,

$\sigma_{i j l}^{\prime}$ : setup time for setting line $l$ up from product $i$ to product $j\left(\sigma_{i j l}^{\prime} \leq C\right.$ and $\left.\sigma_{i j l}^{\prime}=0\right)$,

$\omega_{l 1}$ : setup time for the first slot in line $l$ in macroperiod 1 that has already been performed before macro-period 1 starts (note: if $\omega_{l 1}>0$, then values $x_{j l 1}$ are already known and these variables should be set accordingly),

$I_{j 0}$ : initial inventory of product $j$.

\section{Decision Variables for the Production Lines}

$z_{i j l s}$ : if line $l$ is set up from product $i$ to $j$ at the beginning of slot $s, 0$, otherwise $\left(z_{i j l s} \geq 0\right.$ is sufficient),

$I_{j t}$ : amount of product $j$ in inventory at the end of macro-period $t$,

$q_{j l s}$ : number of products $j$ being produced in line $l$ in slot $s$,

$x_{j l s}=1$, if slot $s$ in line $l$ can be used to produce product $j, 0$, otherwise,

$u_{l s}=1$, if a positive production amount is produced in slot $s$ in production line $l$ (0, otherwise),

$\sigma_{l s}$ : setup time at line $l$ at the beginning of slot $s$,

$\omega_{l t}$ : setup time for the first slot on production line $l$ in macro-period $t$ that is scheduled at the end of macroperiod $t-1$,

$x_{l s \tau}^{E}=1$, if lot $s$ (which belongs to a single macroperiod $t)$ in line $l$ ends in micro-period $\tau$,

$x_{l s \tau}^{B}=1$, if lot $s$ (which belongs to a single macroperiod $t$ ) in line $l$ begins in micro-period $\tau$,

$\delta_{l s}$ : time in the first micro-period of a lot which is reserved for setup time and idle time,

$q_{j}^{0}$ : shortage of product $j$.

\section{Parameters for the Tanks}

$\bar{J}:$ number of raw materials,

$\bar{L}$ : number of parallel tanks,

$\bar{L}_{j}$ : set of tanks in which raw material $j$ can be stored,

$\bar{S}$ : upper bound on the number of lots per macroperiod, that is, the number of slots in tanks,

$\bar{s}_{i j k}$ : sequence-dependent setup cost coefficient for a setup from raw material $i$ to $j$ in tank $l, l\left(\bar{s}_{j j k}\right.$ may be positive),

$\bar{h}_{j}$ : holding cost coefficient for having one unit of raw material $j$ in inventory at the end of a macro-period,

$\bar{v}_{j k}$ : production cost coefficient for filling one unit of raw material $j$ in tank $k$,

$\bar{x}_{j k 0}=1$, if tank $k$ is set up for raw material $j$ at the beginning of the planning horizon,

$\bar{\sigma}_{i j k}^{\prime}$ : setup time for setting tank $k$ up from raw material $i$ to raw material $j\left(\bar{\sigma}_{i j k}^{\prime} \leq C\right)$ (w.l.o.g. $\bar{\sigma}_{i j k}^{\prime}$ is an integer multiple of $C^{m}$ ),

$\bar{\omega}_{k 1}$ : setup time for the first slot in tank $k$ in macroperiod 1 that has already been performed before macro-period 1 starts (note: if $\bar{\omega}_{k 1}>0$ then values $\bar{x}_{j k 1}$ are already known and these variables should be set accordingly),

$\bar{I}_{j k 0}$ : initial inventory of raw material $j$ in tank $k$,

$\bar{Q}_{k}$ : maximum amount to be filled in tank $k$,

$\underline{Q}_{k}$ : minimum amount to be filled in tank $k$.

\section{Decision Variables for the Tanks}

$\bar{z}_{i j k s}=1$, if tank $k$ is set up from raw material $i$ to $j$ at the beginning of slot $s, 0$, otherwise $\left(\bar{z}_{i j k s} \geq 0\right.$ is sufficient),

$\bar{I}_{j k \tau}$ : amount of raw material $j$ in tank $k$ at the end of micro-period $\tau$,

$\bar{q}_{j k s}$ : amount of raw material $j$ being filled in tank $k$ in slot $s$,

$\bar{q}_{j k s \tau}$ : amount of raw material $j$ being filled in tank $k$ in slot $s$ in micro-period $\tau$,

$\bar{x}_{j k s}=1$, if slot $s$ can be used to fill raw material $j$ in tank $k, 0$, otherwise,

$\bar{u}_{k s}=1$, if slot $s$ is used to fill raw material in tank $k$, 0 , otherwise,

$\bar{\sigma}_{k s}$ : setup time, that is, the time for cleaning and filling the tank up, at tank $k$ at the beginning of slot $s$,

$\bar{\omega}_{k t}$ : setup time, that is, the time for cleaning and filling the tank up, for the first slot in tank $k$ in macro-period $t$ that is scheduled at the end of macro-period $t-1$,

$\bar{x}_{k s \tau}^{E}=1$, if lot $s$ at tank $k$ ends in micro-period $\tau$, $\bar{x}_{k s \tau}^{B}=1$, if lot $s$ at tank $k$ begins in micro-period $\tau$. 


\section{Conflict of Interests}

The authors declare that there is no conflict of interests regarding the publication of this paper.

\section{Acknowledgments}

The authors would like to thank the anonymous reviewers for their useful comments and suggestions. They are also indebted to Companhia de Bebidas Ipiranga, Brazil, for its kind collaboration. This research was partially supported by FAPESP and CNPq grants 483474/2013-4 and 312967/2014-4.

\section{References}

[1] G. R. Bitran and H. H. Yanasse, "Computational complexity of the capacitated lot size problem," Management Science, vol. 28, no. 10, pp. 1174-1186, 1982.

[2] W.-H. Chen and J.-M. Thizy, "Analysis of relaxations for the multi-item capacitated lot-sizing problem," Annals of Operations Research, vol. 26, no. 1-4, pp. 29-72, 1990.

[3] J. Maes, J. O. McClain, and L. N. van Wassenhove, "Multilevel capacitated lotsizing complexity and LP-based heuristics," European Journal of Operational Research, vol. 53, no. 2, pp. 131-148, 1991.

[4] K. Haase and A. Kimms, "Lot sizing and scheduling with sequence-dependent setup costs and times and efficient rescheduling opportunities," International Journal of Production Economics, vol. 66, no. 2, pp. 159-169, 2000.

[5] J. R. D. Luche, R. Morabito, and V. Pureza, "Combining process selection and lot sizing models for production scheduling of electrofused grains," Asia-Pacific Journal of Operational Research, vol. 26, no. 3, pp. 421-443, 2009.

[6] A. R. Clark, R. Morabito, and E. A. V. Toso, "Production setupsequencing and lot-sizing at an animal nutrition plant through ATSP subtour elimination and patching," Journal of Scheduling, vol. 13, no. 2, pp. 111-121, 2010.

[7] R. E. Berretta, P. M. França, and V. A. Armentano, "Metaheuristic approaches for the multilevel resource-constrained lot-sizing problem with setup and lead times," Asia-Pacific Journal of Operational Research, vol. 22, no. 2, pp. 261-286, 2005.

[8] T. Wu, L. Shi, J. Geunes, and K. Akartunali, "An optimization framework for solving capacitated multi-level lot-sizing problems with backlogging," European Journal of Operational Research, vol. 214, no. 2, pp. 428-441, 2011.

[9] C. F. M. Toledo, R. R. R. de Oliveira, and P. M. França, "A hybrid multi-population genetic algorithm applied to solve the multi-level capacitated lot sizing problem with backlogging," Computers and Operations Research, vol. 40, no. 4, pp. 910-919, 2013.

[10] C. Almeder, D. Klabjan, R. Traxler, and B. Almada-Lobo, "Lead time considerations for the multi-level capacitated lot-sizing problem," European Journal of Operational Research, vol. 241, no. 3, pp. 727-738, 2015.

[11] A. Kimms, "Demand shuffle-a method for multilevel proportional lot sizing and scheduling," Naval Research Logistics, vol. 44, no. 4, pp. 319-340, 1997.

[12] A. Kimms, Multi-Level Lot Sizing and Scheduling: Methods for Capacitated, Dynamic, and Deterministic Models, Physica, Heidelberg, Germany, 1997.
[13] R. de Matta and M. Guignard, "The performance of rolling production schedules in a process industry," IIE Transactions, vol. 27, pp. 564-573, 1995.

[14] S. Kang, K. Malik, and L. J. Thomas, "Lotsizing and scheduling on parallel machines with sequence-dependent setup costs," Management Science, vol. 45, no. 2, pp. 273-289, 1999.

[15] H. Kuhn and D. Quadt, "Lot sizing and scheduling in semiconductor assembly-a hierarchical planning approach," in Proceedings of the International Conference on Modeling and Analysis of Semiconductor Manufacturing (MASM '02), G. T. Mackulak, J. W. Fowler, and A. Schömig, Eds., pp. 211-216, Tempe, Ariz, USA, 2002.

[16] D. Quadt and H. Kuhn, "Production planning in semiconductor assembly," Working Paper, Catholic University of Eichstätt, Ingolstadt, Germany, 2003.

[17] M. C. V. Nascimento, M. G. C. Resende, and F. M. B. Toledo, "GRASP heuristic with path-relinking for the multi-plant capacitated lot sizing problem," European Journal of Operational Research, vol. 200, no. 3, pp. 747-754, 2010.

[18] A. R. Clark and S. J. Clark, "Rolling-horizon lot-sizing when set-up times are sequence-dependent," International Journal of Production Research, vol. 38, no. 10, pp. 2287-2307, 2000.

[19] L. A. Wolsey, "Solving multi-item lot-sizing problems with an MIP solver using classification and reformulation," Management Science, vol. 48, no. 12, pp. 1587-1602, 2002.

[20] A. R. Clark, "Hybrid heuristics for planning lot setups and sizes," Computers \& Industrial Engineering, vol. 45, no. 4, pp. 545-562, 2003.

[21] D. Gupta and T. Magnusson, "The capacitated lot-sizing and scheduling problem with sequence-dependent setup costs and setup times," Computers \& Operations Research, vol. 32, no. 4, pp. 727-747, 2005.

[22] B. Almada-Lobo, D. Klabjan, M. A. Carravilla, and J. F. Oliveira, "Single machine multi-product capacitated lot sizing with sequence-dependent setups," International Journal of Production Research, vol. 45, no. 20, pp. 4873-4894, 2007.

[23] B. Almada-Lobo, J. F. Oliveira, and M. A. Carravilla, "Production planning and scheduling in the glass container industry: a VNS approach," International Journal of Production Economics, vol. 114, no. 1, pp. 363-375, 2008.

[24] C. F. M. Toledo, M. Da Silva Arantes, R. R. R. De Oliveira, and B. Almada-Lobo, "Glass container production scheduling through hybrid multi-population based evolutionary algorithm," Applied Soft Computing Journal, vol. 13, no. 3, pp. 13521364, 2013.

[25] T. A. Baldo, M. O. Santos, B. Almada-Lobo, and R. Morabito, "An optimization approach for the lot sizing and scheduling problem in the brewery industry," Computers and Industrial Engineering, vol. 72, no. 1, pp. 58-71, 2014.

[26] A. Drexl and A. Kimms, "Lot sizing and scheduling-survey and extensions," European Journal of Operational Research, vol. 99, no. 2, pp. 221-235, 1997.

[27] B. Karimi, S. M. T. Fatemi Ghomi, and J. M. Wilson, "The capacitated lot sizing problem: a review of models and algorithms," Omega, vol. 31, no. 5, pp. 365-378, 2003.

[28] R. Jans and Z. Degraeve, "Meta-heuristics for dynamic lot sizing: a review and comparison of solution approaches," European Journal of Operational Research, vol. 177, no. 3, pp. 1855-1875, 2007.

[29] R. Jans and Z. Degraeve, "Modeling industrial lot sizing problems: a review," International Journal of Production Research, vol. 46, no. 6, pp. 1619-1643, 2008. 
[30] R. Ramezanian, M. Saidi-Mehrabad, and P. Fattahi, "MIP formulation and heuristics for multi-stage capacitated lot-sizing and scheduling problem with availability constraints," Journal of Manufacturing Systems, vol. 32, no. 2, pp. 392-401, 2013.

[31] H. Meyr, "Simultaneous lotsizing and scheduling on parallel machines," European Journal of Operational Research, vol. 139, no. 2, pp. 277-292, 2002.

[32] H. Meyr, "Simultaneous lotsizing and scheduling by combining local search with dual reoptimization," European Journal of Operational Research, vol. 120, no. 2, pp. 311-326, 2000.

[33] S. Li, X. Zhong, and H. Li, "Batch delivery scheduling with multiple decentralized manufacturers," Mathematical Problems in Engineering, vol. 2014, Article ID 321513, 7 pages, 2014.

[34] H. Tempelmeier and L. Buschkühl, "Dynamic multi-machine lotsizing and sequencing with simultaneous scheduling of a common setup resource," International Journal of Production Economics, vol. 113, no. 1, pp. 401-412, 2008.

[35] D. Ferreira, R. Morabito, and S. Rangel, "Solution approaches for the soft drink integrated production lot sizing and scheduling problem," European Journal of Operational Research, vol. 196, no. 2, pp. 697-706, 2009.

[36] D. Ferreira, A. R. Clark, B. Almada-Lobo, and R. Morabito, "Single-stage formulations for synchronised two-stage lot sizing and scheduling in soft drink production," International Journal of Production Economics, vol. 136, no. 2, pp. 255-265, 2012.

[37] C. F. M. Toledo, L. De Oliveira, R. De Freitas Pereira, P. M. França, and R. Morabito, "A genetic algorithm/mathematical programming approach to solve a two-level soft drink production problem," Computers \& Operations Research, vol. 48, pp. 40-52, 2014.

[38] C. F. M. Toledo, P. M. França, R. Morabito, and A. Kimms, "Um modelo de otimização para o problema integrado de dimensionamento de lotes e programação da produção em fábricas de refrigerantes," Pesquisa Operacional, vol. 27, no. 1, pp. 155-186, 2007.

[39] C. F. M. Toledo, P. M. França, R. Morabito, and A. Kimms, "Multi-population genetic algorithm to solve the synchronized and integrated two-level lot sizing and scheduling problem," International Journal of Production Research, vol. 47, no. 11, pp. 3097-3119, 2009. 


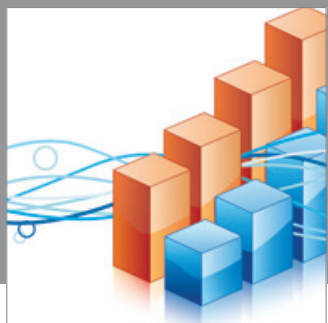

Advances in

Operations Research

mansans

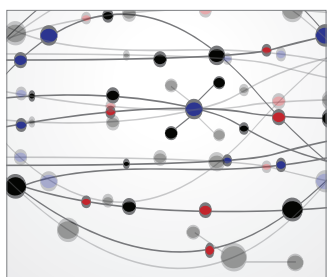

The Scientific World Journal
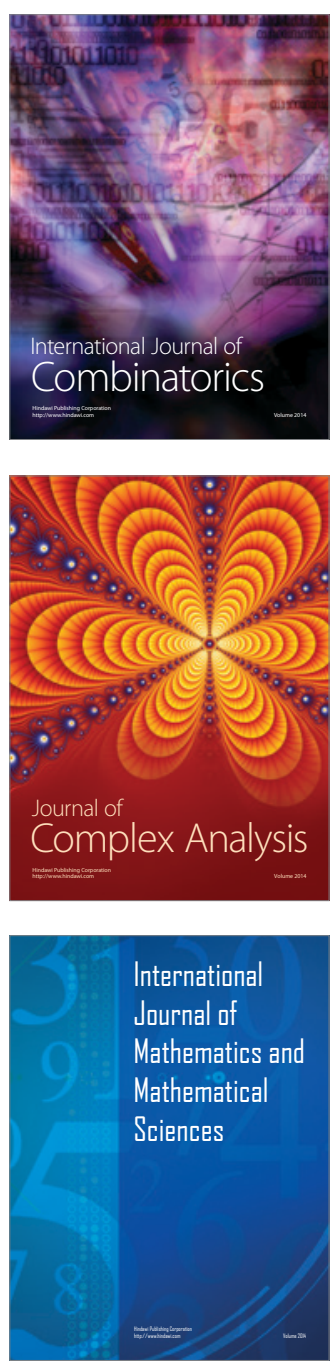
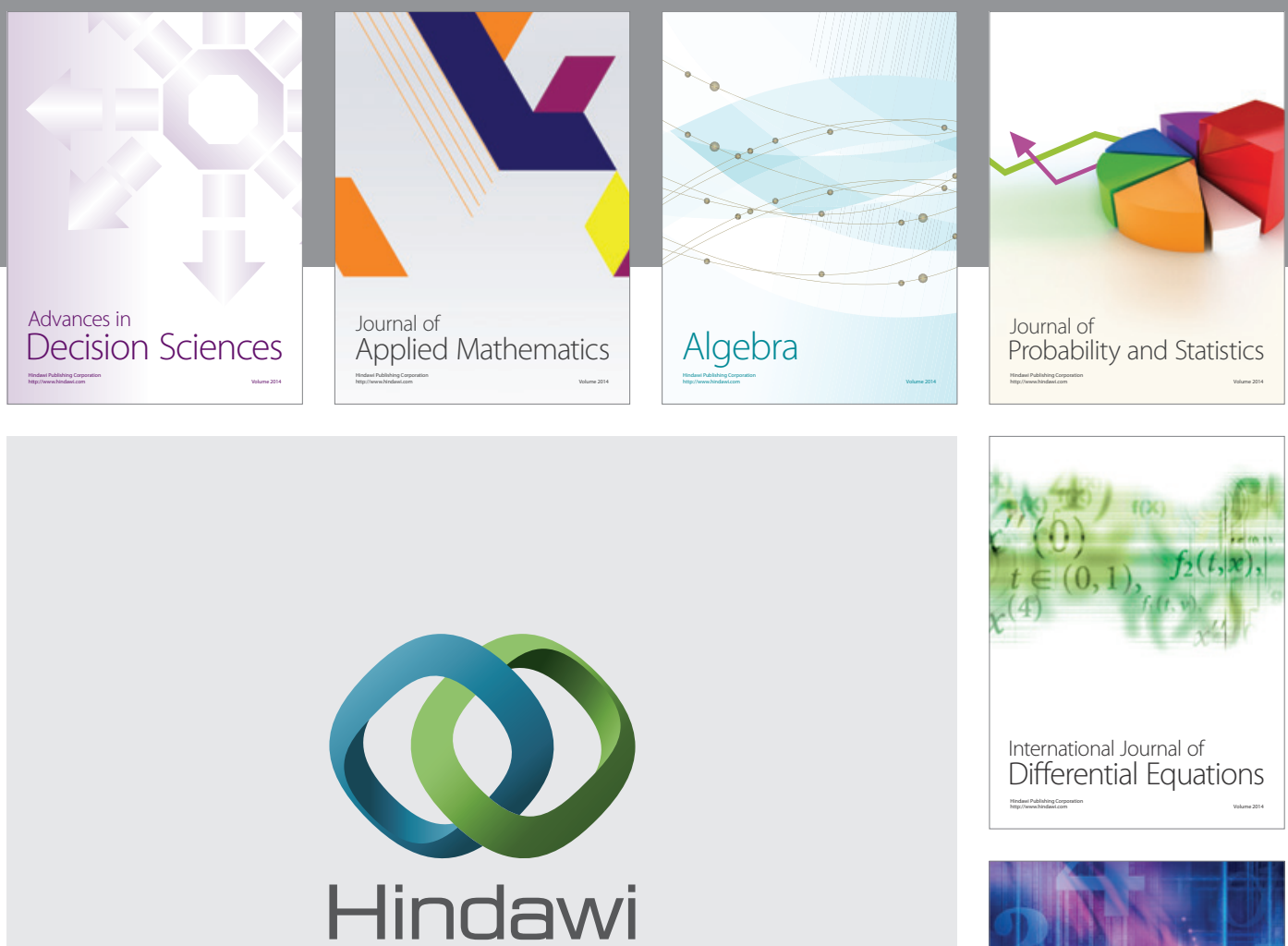

Submit your manuscripts at http://www.hindawi.com
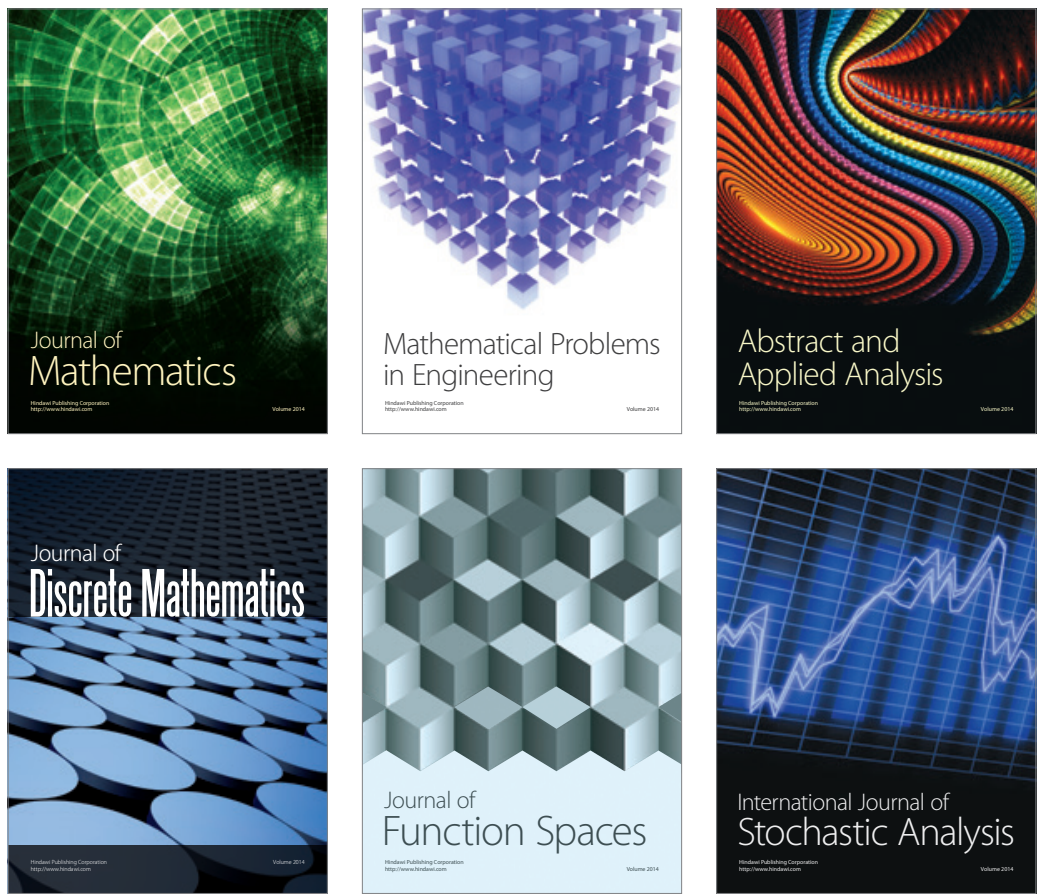

Journal of

Function Spaces

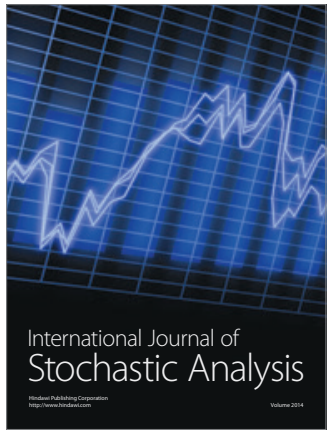

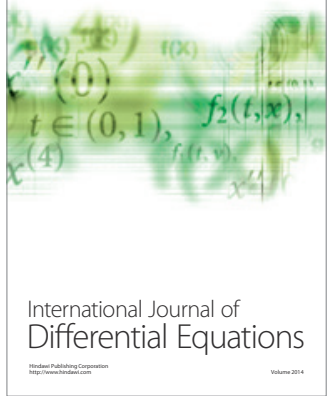
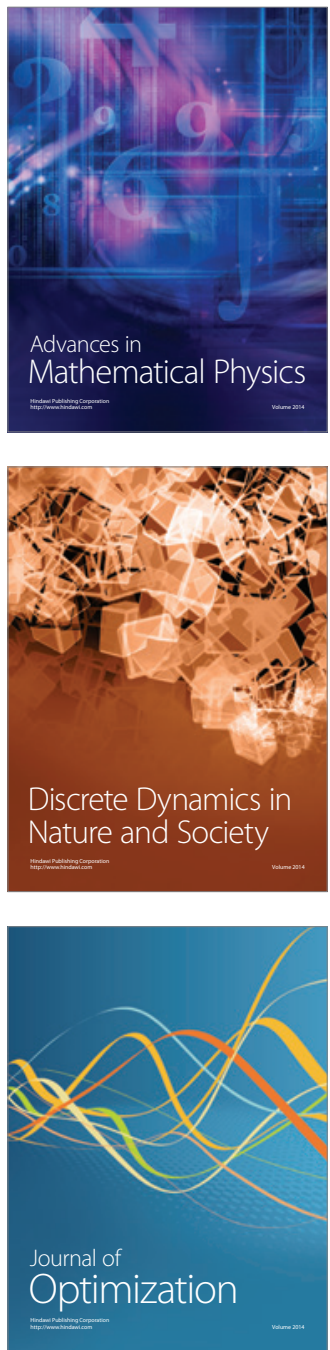